TRANSACTIONS OF THE

AMERICAN MATHEMATICAL SOCIETY

Volume 351, Number 1, January 1999, Pages 41-62

S 0002-9947(99)01827-9

\title{
AN ALGORITHM FOR CALCULATING THE NIELSEN NUMBER ON SURFACES WITH BOUNDARY
}

\author{
JOYCE WAGNER
}

\begin{abstract}
Let $f: M \rightarrow M$ be a self-map of a hyperbolic surface with boundary. The Nielsen number, $N(f)$, depends only on the induced map $f_{\#}$ of the fundamental group, which can be viewed as a free group on $n$ generators, $a_{1}, \ldots, a_{n}$. We determine conditions for fixed points to be in the same fixed point class and if these conditions are enough to determine the fixed point classes, we say that $f_{\#}$ is $W$-characteristic. We define an algebraic condition on the $f_{\#}\left(a_{i}\right)$ and show that "most" maps satisfy this condition and that all maps which satisfy this condition are $W$-characteristic. If $f_{\#}$ is $W$ characteristic, we present an algorithm for calculating $N(f)$ and prove that the inequality $|L(f)-\chi(M)| \leq N(f)-\chi(M)$ holds, where $L(f)$ denotes the Lefschetz number of $f$ and $\chi(M)$ the Euler characteristic of $M$, thus answering in part a question of Jiang and Guo.
\end{abstract}

\section{INTRODUCTION}

Given a self-map, $f$, on a manifold $M$, we wish to obtain information about the fixed point set, $\operatorname{Fix}(f)=\{x \in M \mid f(x)=x\}$. In particular, we are interested in finding the minimum number $M F(f)$ of fixed points among all maps homotopic to $f$, i.e., $M F(f)=\min \{\# \operatorname{Fix}(g) \mid g \sim f\}$.

The Nielsen number, $N(f)$, gives a lower bound for the number of fixed points of $f$, and because of its homotopy invariance, it is a lower bound for $M F(f)$. It is this fact that makes calculation of $N(f)$ a matter of interest.

If $N(f)=M F(f)$, we will say that $f$ is a Wecken map. If all self-maps of $M$ are Wecken, then $M$ is said to be Wecken.

In 1941-42, Wecken published the following result:

Theorem 0.1 ([W]). If $X$ is a compact $n$-manifold where $n \geq 3$, then $X$ is Wecken.

In 1984-85, Jiang [J2] demonstrated that the disc with two holes, also called the pants surface, was not Wecken and was able to modify the example to all surfaces of negative Euler characteristic and obtain

Theorem $0.2([\mathrm{~J} 3])$. A surface is Wecken if and only if its Euler characteristic is non-negative.

Once it was known that hyperbolic surfaces are not Wecken, it became a nontrivial problem to classify those maps where $N(f)=M F[f]$. The following result was proven using Nielsen's and Thurston's work with hyperbolic surfaces.

Received by the editors December 15, 1995.

1991 Mathematics Subject Classification. Primary 55M20.

(C)1999 American Mathematical Society 
Theorem 0.3 ([J4], [JG]). All homeomorphisms of surfaces are Wecken.

In order to answer this classification question, we need to be able to calculate the Nielsen number. By definition, we separate the fixed points of the map $f$ into equivalence classes and assign to each class a number, called the fixed point index. If a class has a nonzero index, we call it essential, otherwise, we say that it is inessential. The Nielsen number, $N(f)$, is then defined to be the number of essential fixed point classes. In most cases, however, calculating $N(f)$ is not easy. McCord surveys many of the existing computational methods in [Mc]. There have also been more recent results, such as the paper by Davey, Hart and Trapp [DHT], which describes an improved method for calculating the Nielsen number of maps on closed surfaces. Our goal in this paper is to develop an algorithm for calculating the Nielsen number of maps of hyperbolic surfaces with boundary for which we only need to know the induced map on the fundamental group.

In Section 1, we convert the condition of being in the same Nielsen class to a condition in the free group isomorphic to $\pi_{1}(M)$. We consider a map $f: C \rightarrow C$ where $C$ is the wedge of $n$ circles, one for each generator of the fundamental group and define $\mathbb{X}$ to be the set of images under $f$ of these generators. In Section 2 , we simplify the set $\mathbb{X}$ and determine a class for which $N(f)=|L(f)|$ where $L(f)$ is the Lefschetz number. In Section 3, we list four conditions which guarantee that two fixed points are in the same class. If those are the only conditions we need to determine the fixed point classes, we say that $f$ is $W$-characteristic. We describe an easily-implemented algorithm for calculating $N(f)$ if $f$ is $W$-characteristic. We define what we mean by $\mathbb{X}$ having remnant and show that most maps have remnants, in a sense that will be made precise in Theorem 3.7. Our main result is

Theorem 3.8. If $f: C \rightarrow C$ is a map such that $\mathbb{X}$ has remnant, then $f$ is $W$ characteristic.

In Section 4, we present the algorithm and in Section 5, we look at an inequality introduced by Jiang and Guo in their paper on homeomorphisms of surfaces [JG] and show that

Theorem 5.1. If $f: C \rightarrow C$ is $W$-characteristic, then $|L(f)-\chi(C)| \leq N(f)-$ $\chi(C)$ where $\chi(C)$ is the Euler characteristic.

In particular, if $\mathbb{X}$ has remnant, then the inequality holds. Since the Lefschetz number, the Euler characteristic and the Nielsen number are homotopy and homotopy-type invariant, we have

Corollary 5.2. If $f: M \rightarrow M$ is a self-map of a hyperbolic surface with boundary and the induced map of the fundamental group is $W$-characteristic, then

$$
|L(f)-\chi(M)| \leq N(f)-\chi(M) .
$$

Readers who just wish to know how to calculate Nielsen numbers need only read Section 4, noting any earlier definitions as indicated. For more background information on the Nielsen number see $[\mathrm{B}],[\mathrm{J} 1],[\mathrm{Ki}]$ and $[\mathrm{N}]$.

This paper was submitted as partial fulfillment of the requirements for the Ph.D. at the University of California at Los Angeles under the supervision of Professor Robert Brown. I wish to thank Professor Brown for his invaluable assistance and Professor Helga Schirmer for her many helpful comments. 


\section{WHEN FIXED POINTS ARE IN THE SAME CLASS}

Suppose $f: M \rightarrow M$ is a self-map of a hyperbolic surface with boundary. Given a point $x_{0}$, the fundamental group $\pi_{1}\left(M, x_{0}\right)$ can be represented by the union $C$ of simple closed oriented curves, $C_{1}, \ldots, C_{n}$ in $M$, which meet only at the point $x_{0}$. Therefore, we can represent the induced map $f_{\#}$ of the fundamental group by a map $f_{\#}: C \rightarrow C$. Since $M$ is homotopy equivalent to $C$ and the Nielsen number is a homotopy-type invariant [J1], then $N(f)=N\left(f_{\#}\right)$ and so we need only consider self-maps of $C$. We identify $\pi_{1}\left(M, x_{0}\right)$ with the free group $G$ on the letters $a_{i}$, $1 \leq i \leq n$, by associating the homotopy class of each oriented loop $C_{i}$ with $a_{i}$. For simplicity of notation, we will identify any $z \in \pi_{1}\left(M, x_{0}\right)$ with its counterpart in $G$.

Thus, let $f: C \rightarrow C$ be any map. Because $N(f)$ is homotopy invariant, we may assume that we have chosen $f$ so that $f\left(a_{i}\right)=X_{i}$ is a reduced word in $G$. We can also assume that $f(D)=x_{0}$ where $D$ is the closure of a small neighborhood of $x_{0}$. The components of the complement of $D$ are mapped onto $C$ in the manner determined by the $X_{i}$. We can think of these complementary regions as being divided into equal intervals, where the interior of each interval is mapped homeomorphically onto $C_{i}-x_{0}$ for some $i$.

By construction, there is a fixed point in each interval in $C_{i}$ that is sent onto itself, that is, for every $a_{i}$ of $a_{i}^{-1}$ that appears in $X_{i}$. The only other fixed point will be $x_{0}$. Notice that there can only be a finite number of fixed points.

We wish to determine when two fixed points $x_{1}$ on $C_{i_{1}}$ and $x_{2}$ on $C_{i_{2}}$ are in the same fixed point class. Let $\gamma_{j}^{+}$and $\gamma_{j}^{-}$denote the arcs of $C_{i_{j}}$ going from $x_{0}$ to $x_{j}$ in the positive and negative directions, respectively. The fact that $\gamma_{j}^{+}\left(\gamma_{j}^{-}\right)^{-1}=a_{i_{j}}$ gives us:

Lemma 1.1. The arcs $\gamma_{j}^{+}$and $\gamma_{j}^{-}$satisfy the following homotopy relations:

$$
\gamma_{j}^{-} \sim a_{i_{j}}^{-1} \gamma_{j}^{+} \text {and }\left(\gamma_{j}^{-}\right)^{-1} \sim\left(\gamma_{j}^{+}\right)^{-1} a_{i_{j}} \text { rel } x_{0} .
$$

Consequently, any path $\gamma$ connecting $x_{1}$ and $x_{2}$ can be written in the form

$$
\gamma=\left(\gamma_{1}^{+}\right)^{-1} z \gamma_{2}^{+}
$$

where $z \in \pi_{1}\left(M, x_{0}\right)$ and is therefore identified with $z \in G$.

In the special case where $x_{1}=x_{0}$, we write $\gamma$ in the form

$$
\gamma=z \gamma_{2}^{+} \text {. }
$$

By definition, the fixed points $x_{1}$ and $x_{2}$ are in the same class if and only if there exists a path $\gamma$ connecting the two fixed points such that $f(\gamma) \sim \gamma$ rel endpoints. By Lemma 1.1,

$$
f(\gamma)=f\left(\left(\gamma_{1}^{+}\right)^{-1}\right) f(z) f\left(\gamma_{2}^{+}\right) .
$$

To convert the relation determining fixed point classes into a condition in the free group, we would like to be able to write $f\left(\gamma_{j}^{+}\right)=W_{j} \gamma_{j}^{+}$and $f\left(\gamma_{j}^{-}\right)=\bar{W}_{j} \gamma_{j}^{-}$ where $W_{j}$ and $\bar{W}_{j}$ are in $G$. We do that as follows:

Lemma 1.2. Suppose that $x_{j}$ is the fixed point corresponding to the occurrence of $a_{i_{j}}^{\varepsilon_{j}}$ in $f\left(a_{i_{j}}\right)=V_{j} a_{i_{j}}^{\varepsilon_{j}} \bar{V}_{j}$ where $\varepsilon_{j}=\{+1,-1\}$. If we write $f\left(\gamma_{j}^{+}\right)=W_{j} \gamma_{j}^{+}$, then

$$
W_{j}= \begin{cases}V_{j} & \text { if } \varepsilon_{j}=1, \\ V_{j} a_{i_{j}}^{-1} & \text { if } \varepsilon_{j}=-1 .\end{cases}
$$


If we write $f\left(\gamma_{j}^{-}\right)=\bar{W}_{j} \gamma_{j}^{-}$, then

$$
\bar{W}_{j}= \begin{cases}\bar{V}_{j}^{-1} & \text { if } \varepsilon_{j}=1, \\ \bar{V}_{j}^{-1} a_{i_{j}} & \text { if } \varepsilon_{j}=-1 .\end{cases}
$$

If $x_{j}=x_{0}$, then $W_{j}=\bar{W}_{j}=1$.

Proof. Suppose first that $\varepsilon_{j}=1$, then

$$
f\left(\gamma_{j}^{+}\right) f\left(\left(\gamma_{j}^{-}\right)^{-1}\right)=f\left(a_{i_{j}}\right)=V_{j} a_{i_{j}} \bar{V}_{j}=V_{j} \gamma_{j}^{+}\left(\gamma_{j}^{-}\right)^{-1} \bar{V}_{j} .
$$

Since $f\left(x_{j}\right)=x_{j}$, we conclude that

$$
W_{j} \gamma_{j}^{+}=f\left(\gamma_{j}^{+}\right)=V_{j} \gamma_{j}^{+}
$$

and

$$
\bar{W}_{j} \gamma_{j}^{-}=f\left(\gamma_{j}^{-}\right)=\bar{V}_{j}^{-1} \gamma_{j}^{-} .
$$

Therefore $W_{j}=V_{j}$ and $\bar{W}_{j}=\bar{V}_{j}^{-1}$.

Suppose now that $\varepsilon_{j}=-1$, then

$$
f\left(\gamma_{j}^{+}\right) f\left(\left(\gamma_{j}^{-}\right)^{-1}\right)=f\left(a_{i_{j}}\right)=V_{j} a_{i_{j}}^{-1} \bar{V}_{j}=V_{j} \gamma_{j}^{-}\left(\gamma_{j}^{+}\right)^{-1} \bar{V}_{j} .
$$

Since $f\left(x_{j}\right)=x_{j}$, we conclude that

$$
W_{j} \gamma_{j}^{+}=f\left(\gamma_{j}^{+}\right)=V_{j} \gamma_{j}^{-}
$$

and

$$
\bar{W}_{j} \gamma_{j}^{-}=f\left(\gamma_{j}^{-}\right)=\bar{V}_{j}^{-1} \gamma_{j}^{+} .
$$

Therefore $W_{j}=V_{j} \gamma_{j}^{-}\left(\gamma_{j}^{+}\right)^{-1}=V_{j} a_{i_{j}}^{-1}$ and $\bar{W}_{j}=\bar{V}_{j}^{-1} \gamma_{j}^{+}\left(\gamma_{j}^{-}\right)^{-1}=\bar{V}_{j}^{-1} a_{i_{j}}$.

If $x_{j}=x_{0}$, then $\gamma_{j}^{+}=\gamma_{j}^{-}=x_{0}$. Since $f\left(x_{0}\right)=x_{0}$, then $f\left(\gamma_{j}^{+}\right)=\gamma_{j}^{+}$and $f\left(\gamma_{j}^{-}\right)=\gamma_{j}^{-}$, so $W_{j}=\bar{W}_{j}=1$.

Lemma 1.3. We can write $f\left(a_{i_{j}}\right)=W_{j} a_{i_{j}} \bar{W}_{j}^{-1}$, where $W_{j}$ and $\bar{W}_{j}$ are defined as in Lemma 1.2.

Proof. From Lemma 1.2 we have

$$
\begin{aligned}
f\left(a_{i_{j}}\right) & =f\left(\gamma_{j}^{+}\right) f\left(\left(\gamma_{j}^{-1}\right)^{-1}\right) \\
& =\left(W_{j} \gamma_{j}^{+}\right)\left(\left(\gamma_{j}^{-}\right)^{-1} \bar{W}_{j}^{-1}\right)=W_{j} a_{i_{j}} \bar{W}_{j}^{-1} .
\end{aligned}
$$

Note that the definition of $W_{j}$ and $\bar{W}_{j}$ depends on the exponent $\varepsilon_{j}$ that is associated with the fixed point. Similarly, the fixed point index also depends on the exponent as follows:

$$
i\left(f, x_{j}\right)= \begin{cases}-1 & \text { if } \varepsilon_{j}=1, \\ +1 & \text { if } \varepsilon_{j}=-1 \quad \text { or } \quad x_{j}=x_{0} .\end{cases}
$$

The index $i\left(F_{i}\right)$ of a fixed point class $F_{i}$ is defined to be the sum of the indexes of all the fixed points in $F_{i}$. For the general definition of index, see [B] or [J1].

Example 1.4. If $X_{1}=a_{2} a_{1} a_{3}^{-2} a_{1}^{-1}$, then there are two fixed points on the loop $C_{1}$. The first fixed point corresponds to an occurrence of $a_{1}$ and therefore $i\left(f, x_{1}\right)=-1$, $W_{1}=a_{2}$ and $\bar{W}_{1}=a_{1} a_{3}^{2}$. The second fixed point corresponds to an occurrence of $a_{1}^{-1}$ and therefore $i\left(f, x_{2}\right)=1, W_{2}=a_{2} a_{1} a_{3}^{-2} a_{1}^{-1}$ and $\bar{W}_{2}=a_{1}$. 
We now have the following necessary and sufficient condition for two fixed points to belong to the same fixed point class.

Lemma 1.5. Two fixed points $x_{1}$ and $x_{2}$ are in the same fixed point class if and only if there is a solution $z$ to the equation in $G$ :

$$
z=W_{1}^{-1} f(z) W_{2} .
$$

Proof. Recall that the two fixed points are in the same fixed point class if and only if there exists a path $\gamma=\left(\gamma_{1}^{+}\right)^{-1} z \gamma_{2}^{+}$such that $f(\gamma) \sim \gamma$ rel endpoints. From Lemma 1.1 and the definitions of the $W_{i}$, we have that

$$
\begin{aligned}
f(\gamma) & =f\left(\left(\gamma_{1}^{+}\right)^{-1}\right) f(z) f\left(\gamma_{2}^{+}\right) \\
& =\left(\gamma_{1}^{+}\right)^{-1} W_{1}^{-1} f(z) W_{2}\left(\gamma_{2}^{+}\right) .
\end{aligned}
$$

Our goal, therefore, is to determine if there can exist such a $z \in G$, the free group on the $a_{i}$.

\section{Simplifications}

Given our map $f$, we look at the corresponding set of words $\mathbb{X}=\left\{X_{1}, \ldots, X_{n}\right\}$ and perform the following simplifications.

(S1) If there is a word $U \neq 1$ in $G$ such that $X_{i}=U X_{i}^{\prime} U^{-1}, X_{i}=U^{ \pm 1}$ or $X_{i}=1$ for all $i$ and there is at least one $X_{j}=U X_{j}^{\prime} U^{-1}$, then replace each $X_{i}$ by $X_{i}^{\prime}=U^{-1} X_{i} U$. The corresponding map $f^{\prime}$ will be homotopic to $f$ and so $N(f)=N\left(f^{\prime}\right)$ since the Nielsen number is a homotopy invariant [J1].

(S2) Suppose two or more of the $X_{i}$ are powers of a word $U$, then renumber the $a_{i}$ so that $X_{i}=U^{r_{i}}$ for $m \leq i \leq n$. Let $E$ be the bouquet of $m$ circles and recall that $C$ was the bouquet of $n$ circles. We will use the commutativity property of the Nielsen number which states that if $h: E \rightarrow C$ and $g: C \rightarrow E$ are maps of finite polyhedra, then $N(g \circ h)=N(h \circ g)$ [J1]. We define maps $h, g$ so that for $b_{1}, \ldots, b_{m}$ generating $\pi_{1}(E)$, we have

$$
\begin{aligned}
h_{\#}\left(b_{i}\right) & = \begin{cases}X_{i}, & 1 \leq i \leq m-1, \\
U, & i=m,\end{cases} \\
g_{\#}\left(a_{i}\right) & = \begin{cases}b_{i}, & 1 \leq i \leq m-1, \\
b_{m}^{r_{i}}, & m \leq i \leq n\end{cases}
\end{aligned}
$$

Since $h_{\#} g_{\#}=f_{\#}$ and $C$ is a $K(\pi, 1)$, the map $f$ is homotopic to $h \circ g$ and thus $N(f)=N(h \circ g)=N(g \circ h)$. Now, $g \circ h: E \rightarrow E$ is a map such that

$$
g_{\#} h_{\#}\left(b_{i}\right)= \begin{cases}g_{\#}\left(X_{i}\right), & 1 \leq i \leq m-1, \\ g_{\#}(U), & i=m .\end{cases}
$$

To simplify the notation, we will let each $g_{\#} h_{\#}\left(b_{i}\right)=X_{i}$ and $a_{i}=b_{i}$, and we are now reduced to considering $X_{1}, \ldots, X_{m}$ in the free group $G$ on $a_{1}, \ldots, a_{m}$.

We will stop when we can no longer apply (S1) or (S2).

Theorem 2.1. If, at some point in the simplification process, $X_{i}=U^{r_{i}}$ for all $i$, then $N(f)=|L(f)|$. 
Proof. We can apply (S2) with $m=1$. Thus $g \circ h: S^{1} \rightarrow S^{1}$ and $g_{\#} h_{\#}\left(b_{1}\right)=g_{\#}(U)$. If $\alpha_{i}(U)$ denotes the sum of the exponents of all the $a_{i}^{\varepsilon}$ occurring in $U$, then

$$
g_{\#}(U)=b_{1}^{\sum_{1 \leq i \leq n} r_{i} \alpha_{i}(U)} .
$$

Since $g \circ h$ is a self-map of the circle having degree $\sum_{1 \leq i \leq n} r_{i} \alpha_{i}(U)$, then by [B] (p. 107),

$$
N\left(g_{\#}\right)=\left|1-\operatorname{degree}\left(g_{\#}\right)\right|=\left|1-\sum_{1 \leq i \leq n} r_{i} \alpha_{i}(U)\right|
$$

which is equal to $|L(f)|$.

Example 2.2. Let us consider the following example of Weier [We].

$$
X_{1}=a_{1}, \quad X_{2}=a_{4}^{-1} a_{2} a_{4}, \quad X_{3}=a_{3}, \quad X_{4}=1 .
$$

We note that $X_{3}=a_{3}$ and we may write $X_{4}=a_{3}^{0}$. Therefore, we apply (S2) with $U=a_{3}$. Let $h_{\#}\left(b_{i}\right)=X_{i}$ for $1 \leq i \leq 3$ and

$$
g_{\#}\left(a_{i}\right)= \begin{cases}b_{i} & \text { if } 1 \leq i \leq 3 \\ 1 & \text { if } i=4\end{cases}
$$

We then look at the set $g_{\#} h_{\#}\left(b_{i}\right)=g_{\#}\left(X_{i}\right)$ for $1 \leq i \leq 3$ to obtain the new set of words

$$
X_{1}=a_{1}, \quad X_{2}=a_{2}, \quad X_{3}=a_{3} .
$$

Since this set represents the identity map and $L(f)=-2 \neq 0$, then $N(f)=1$.

\section{REMNANTS AND $W$-CHARACTERISTIC MAPS}

The following is a list of sufficient conditions on the words $W_{j}$ and $\bar{W}_{j}$ in $G$ for two fixed points to be in the same fixed point class.

Theorem 3.1. Two fixed points $x_{1}$ and $x_{2}$ are in the same fixed point class if one of the following occurs:

[1.1] $W_{1}=W_{2}$.

[1.2] $W_{1}=\bar{W}_{2}$ or $\bar{W}_{1}=W_{2}$.

[1.3] $\bar{W}_{1}=\bar{W}_{2}$.

Proof. If we let

$$
T=W_{1}^{-1} f(z) W_{2},
$$

then in each case we need to find a $z$ such that $T=z$.

(1) Take $z=1$, then

$$
T=W_{1}^{-1} W_{2}=1=z .
$$

(2) The condition is symmetric, so we take $W_{1}=\bar{W}_{2}$ and let $z=a_{2}^{-1}$. By Lemma 1.3 ,

$$
T=W_{1}^{-1} f\left(a_{2}^{-1}\right) W_{2}=W_{1}^{-1}\left(\bar{W}_{2} a_{2}^{-1} W_{2}^{-1}\right) W_{2}=a_{2}^{-1}=z .
$$


(3) Let $z=a_{1} a_{2}^{-1}$. By Lemma 1.3,

$$
\begin{aligned}
T & =W_{1}^{-1} f\left(a_{1}\right) f\left(a_{2}^{-1}\right) W_{2} \\
& =W_{1}^{-1}\left(W_{1} a_{1} \bar{W}_{1}^{-1}\right)\left(\bar{W}_{2} a_{2}^{-1} W_{2}^{-1}\right) W_{2} \\
& =a_{1}\left(\bar{W}_{1}^{-1} \bar{W}_{2}\right) a_{2}^{-1}=a_{1} a_{2}^{-1}=z .
\end{aligned}
$$

Definitions 3.2. If two fixed points $x_{1}$ and $x_{2}$ satisfy [1.1], [1.2] or [1.3] of Theorem 3.1, then we say that $x_{1}$ and $x_{2}$ are directly related. Suppose that $x_{1}=$ $y_{0}, y_{1}, \ldots, y_{m}, y_{m+1}=x_{2}$ are fixed points. We say that $y_{1}, \ldots, y_{m}$ are intermediate fixed points for $x_{1}$ and $x_{2}$ if $y_{i}$ and $y_{i+1}$ are directly related for each $0 \leq i \leq m$. We also say that $x_{1}$ and $x_{2}$ are related by intermediate fixed points. Note that if $x_{1}$ and $x_{2}$ are directly related, then they are also related by intermediate fixed points with $m=0$. If any two fixed points which are in the same fixed point class are related by intermediate fixed points, then we say that $f$ is $W$-characteristic.

Notice that if $f$ is $W$-characteristic, then a fixed point $x_{1}$ in a fixed point class $F$ must be directly related to at least one other fixed point in $F$ or $F=\left\{x_{1}\right\}$. Therefore, using $W_{i}$ and $\bar{W}_{i}$, we can determine the fixed point classes and thus calculate $N(f)$. An example of the algorithm is presented in Section 4.

We will denote the remainder of this section to defining a certain class of maps $f: C \rightarrow C$ and proving that all such maps are $W$-characteristic.

Write $z=a_{j_{1}}^{\alpha_{1}} a_{j_{2}}^{\alpha_{2}} \cdots a_{j_{m}}^{\alpha_{m}}$ where each $1 \leq j_{i} \leq m$ and $j_{i} \neq j_{i}+1$ for any $i$. Let $\Phi_{a_{i}}(z)$ be the number of occurrences of $a_{i}$ or $a_{i}^{-1}$ in $z$ and let $A=\sum_{1<i<m} \Phi_{a_{i}}(z)$. Therefore, $|z|=A$ where $|W|$ denotes the length of the reduced form of a word $W$.

Given $z$ as above, we have that

$$
f(z)=X_{j_{1}}^{\alpha_{1}} X_{j_{2}}^{\alpha_{2}} \cdots X_{j_{m}}^{\alpha_{m}}
$$

where the right hand side of the equality is not necessarily reduced. The reduced form of $f(z)$, and thus $|f(z)|$, depend on the relationships among the $X_{i}$.

Definitions 3.3. Suppose $\mathbb{S}$ is a set of reduced words in the free group $G$ and $T \in \mathbb{S}^{ \pm 1}$ where $\mathbb{S}^{ \pm 1}$ is the set of words in $\mathbb{S}$ and their inverses. Let $M(T, Z)$ be the longest initial segment of $T$ that cancels in the product $Z^{-1} T$ for $Z \in \mathbb{S}^{ \pm 1}$. Let $M(T, \mathbb{S})$ be the longest of all the $M(T, Z)$ where $Z \neq T$. Then, by definition, $M\left(T^{-1}, \mathbb{S}\right)^{-1}$ will be the longest terminal segment of $T$ that cancels in any product $T Z$ for $Z \in \mathbb{S}^{ \pm 1}$. Therefore, we can write $T=M(T, \mathbb{S}) T^{\prime}=T^{\prime \prime} M\left(T^{-1}, \mathbb{S}\right)^{-1}$. If we can write $T=M(T, \mathbb{S}) \bar{T} M\left(T^{-1}, \mathbb{S}\right)^{-1}$ where both sides of the equation are reduced and $\bar{T} \neq 1$, then we call $\bar{T}$ the remnant of $T$ in $\mathbb{S}$. If every element in $\mathbb{S}$ has a remnant, then we say that $\mathbb{S}$ has remnant. Let

$$
M(\mathbb{S})=\left\{M(T, \mathbb{S})^{\varepsilon} \neq 1 \mid T \in \mathbb{S}^{ \pm 1}, \varepsilon= \pm 1\right\} .
$$

We will call any element in $M(\mathbb{S})$ a maximal common factor or MCF.

In our particular case, we are interested in the set of words $\mathbb{X}=\left\{X_{1}, \ldots, X_{n}\right\}$ in $G$ and we say that $f$ has remnant if $\mathbb{X}$ has remnant. We will set $P_{i}=M\left(X_{i}, \mathbb{X}\right)$ and $S_{i}=M\left(X_{i}^{-1}, \mathbb{X}\right)^{-1}$. 
Example 3.4. Suppose $\mathbb{X}=\left\{X_{1}, X_{2}, X_{3}, X_{4}\right\}$ as follows:

$$
\begin{aligned}
& X_{1}=a_{1}^{2} a_{2}^{2} a_{1}^{-1} a_{3}^{-1}, \\
& X_{2}=a_{1}^{2} a_{2} a_{3}^{-1}, \\
& X_{3}=a_{3} a_{2}^{-3} a_{1}^{-2}, \\
& X_{4}=a_{1}^{-3} .
\end{aligned}
$$

To determine $P_{1}=M\left(X_{1}, \mathbb{X}\right)$, we find $M\left(X_{1}, X_{j}^{\varepsilon}\right)$ for every $X_{j}^{\varepsilon} \neq X_{1}$. Since $a_{1}^{2} a_{2}$ is the longest initial segment of $X_{1}$ that cancels in

$$
X_{2}^{-1} X_{1}=\left(a_{3} a_{2}^{-1} a_{1}^{-2}\right)\left(a_{1}^{2} a_{2}^{2} a_{1}^{-1} a_{3}^{-1}\right)
$$

then $M\left(X_{1}, X_{2}\right)=a_{1}^{2} a_{2}$. We can also see that

$$
\begin{aligned}
M\left(X_{1}, X_{3}\right)=1, & M\left(X_{1}, X_{2}^{-1}\right)=1, \\
M\left(X_{1}, X_{4}\right)=1, & M\left(X_{1}, X_{3}^{-1}\right)=a_{1}^{2} a_{2}^{2}, \\
M\left(X_{1}, X_{1}^{-1}\right)=1, & M\left(X_{1}, X_{4}^{-1}\right)=a_{1}^{2}
\end{aligned}
$$

and therefore $P_{1}=a_{1}^{2} a_{2}^{2}$.

To determine $S_{1}=M\left(X_{1}^{-1}, \mathbb{X}\right)^{-1}$ we find $M\left(X_{1}^{-1}, X_{j}^{\varepsilon}\right)$ for every $X_{j}^{\varepsilon} \neq X_{1}^{-1}$. Since there is no cancelation in the product $X_{1}^{-1} X_{1}^{-1}=\left(a_{3} a_{1} a_{2}^{-2} a_{1}^{-2}\right)\left(a_{3} a_{1} a_{2}^{-2} a_{1}^{-2}\right)$, then $M\left(X_{1}^{-1}, X_{1}\right)=1$. Similarly,

$$
\begin{array}{ll}
M\left(X_{1}^{-1}, X_{2}\right)=1, & M\left(X_{1}^{-1}, X_{2}^{-1}\right)=a_{3} \\
M\left(X_{1}^{-1}, X_{3}\right)=a_{3}, & M\left(X_{1}^{-1}, X_{3}^{-1}\right)=1 \\
M\left(X_{1}^{-1}, X_{4}\right)=1, & M\left(X_{1}^{-1}, X_{4}^{-1}\right)=1
\end{array}
$$

and so $M\left(X_{1}^{-1}, \mathbb{X}\right)=a_{3}$. Therefore, $S_{1}=a_{3}^{-1}$ and $X_{1}=\left[a_{1}^{2} a_{2}^{2}\right] a_{1}^{-1}\left[a_{3}^{-1}\right]=P_{1} a_{1}^{-1} S_{1}$. By Definition 3.3, $X_{1}$ has a remnant.

We do the same for the other $X_{i}$ to get that

$$
\begin{array}{ll}
P_{2}=a_{1}^{2} a_{2}, & S_{2}=a_{2} a_{3}^{-1}, \\
P_{3}=a_{3} a_{2}^{-1}, & S_{3}=a_{2}^{-2} a_{1}^{-2}, \\
P_{4}=1, & S_{4}=a_{1}^{-2},
\end{array}
$$

and therefore

$$
\begin{aligned}
& X_{2}=P_{2} a_{3}^{-1}=a_{1}^{2} S_{2}, \\
& X_{3}=P_{3} S_{3}, \\
& X_{4}=a_{1}^{-1} S_{4} .
\end{aligned}
$$

By Definition 3.3, $X_{4}$ has a remnant, but $X_{2}$ and $X_{3}$ do not, and so $\mathbb{X}$ does not have remnant.

Lemma 3.5. If $\mathbb{X}$ has remnant and $z=a_{i_{1}}^{\varepsilon_{1}} \cdots a_{i_{k}}^{\varepsilon_{k}}$ is a non-trivial, reduced word in $G$, where $\varepsilon_{j} \in\{-1,+1\}$, then we can write $f(z)=U R_{1} \cdots R_{k} V^{-1}$ where

(1) $U R_{1} \cdots R_{k} V^{-1}$ is reduced.

(2) For all $j, R_{j}^{\varepsilon_{j}}$ is a non-trivial subword of $X_{i_{j}}$.

(3) $U=M\left(X_{i_{1}}^{\varepsilon_{1}}, \mathbb{X}\right)$ and $V=M\left(X_{i_{k}}^{-\varepsilon_{k}}, \mathbb{X}\right)$.

(4) $|f(z)| \geq|z|$. 
Proof. Since $z=a_{i_{1}}^{\varepsilon_{1}} \cdots a_{i_{k}}^{\varepsilon_{k}}$, then $f(z)=X_{i_{1}}^{\varepsilon_{1}} \cdots X_{i_{k}}^{\varepsilon_{k}}$ and therefore, by Definition $3.3, f(z)=\left(P_{i_{1}}, \bar{X}_{i_{1}}, S_{i_{1}}\right)^{\varepsilon_{1}} \cdots\left(P_{i_{k}} \bar{X}_{i_{k}} S_{i_{k}}\right)^{\varepsilon_{k}}$. Since $P_{i_{j}}$ and $S_{i_{j}}$ are the maximal portions of $X_{i_{j}}$ that can cancel in a product of the $X_{i}$ 's, then $\bar{X}_{i_{j}}^{\varepsilon_{j}}$ will remain uncancelled. If we let $U=M\left(X_{i_{1}}^{\varepsilon_{1}}, \mathbb{X}\right)$ and $V=M\left(X_{k}^{-\varepsilon_{k}}, \mathbb{X}\right)$ and $R_{j}$ be the portion of $X_{i_{j}}^{\varepsilon_{j}}$ that remains uncancelled in $U^{-1} f(z) V$, then $R_{j}^{\varepsilon_{j}}$ contains $\bar{X}_{i_{j}}$ and so $R_{j}$ is non-trivial. This implies that

$$
|f(z)| \geq \sum_{1 \leq j \leq k}\left|R_{j}\right| \geq k=|z| .
$$

Using Lemma 3.5, we can show that if $\mathbb{X}$ has remnant, then the set $f^{k}(\mathbb{X})=$ $\left\{f^{k}\left(X_{1}\right), \ldots, f^{k}\left(X_{n}\right)\right\}$ also has remnant for all $k \geq 1$. It is enough to prove

Lemma 3.6. If $\mathbb{X}$ has remnant, then $f(\mathbb{X})$ has remnant.

Proof. Suppose that $X_{i} \in \mathbb{X}$. Let $Y$ and $Z$ be any elements in $\mathbb{X}^{ \pm 1}$ and write $X_{i}=A B C, Y=A D$ and $Z=E C$, where $A=M\left(X_{i}, Y\right)$ and $C=M\left(X_{i}^{-1}, Z^{-1}\right)^{-1}$. It is possible that $A$ and $C$ are trivial, but since $\mathbb{X}$ has remnant, then $B, D$ and $E$ are non-trivial. We have that $f\left(X_{i}\right)=f(A) f(B) f(C), f(Y)=f(A) f(D)$ and $f(Z)=$ $f(E) f(C)$. By Lemma 3.5(1), we can write $f(B)=U R_{1} \cdots R_{k} V^{-1}$. Since $B_{s} \neq$ $D_{s}$, then $U$ is the longest initial segment of $f(B)$ that will cancel in the product $f(D)^{-1} f(B)$ and so $M\left(f\left(X_{i}\right), f(Y)\right)$ is at most $f(A) U$. Similarly, since $B_{e} \neq E_{e}$, then $M\left(f\left(X_{i}\right)^{-1}, f(Z)^{-1}\right)^{-1}$ is at most $V^{-1} f(C)$. Since $Y$ and $Z$ were arbitrary elements, then $M\left(f\left(X_{i}\right), f(\mathbb{X})\right)$ is at most $f(A) U$ and $M\left(f\left(X_{i}\right)^{-1}, f(\mathbb{X})\right)^{-1}$ is at most $V^{-1} f(C)$. Thus

$$
f\left(X_{i}\right)=M\left(f\left(X_{i}\right), f(\mathbb{X})\right) \overline{f\left(X_{i}\right)} M\left(f\left(X_{i}\right)^{-1}, f(\mathbb{X})\right)^{-1}
$$

where $\overline{f\left(X_{i}\right)}$ contains $R_{1} \cdots R_{k}$ which is non-trivial by Lemma 3.5(2). Therefore, $f\left(X_{i}\right)$ has a remnant for all $i$, and so $f(\mathbb{X})$ has remnant.

It can also be shown that 'most' maps of hyperbolic surfaces with boundary have remnants. The following theorem and proof were suggested by Professor Robert Brown.

Theorem 3.7. Given $\varepsilon>0$, there exists $M>0$ such that if $\mathbb{X}=\left\{X_{1}, \ldots, X_{n}\right\}$ is chosen at random from among all words of length $\leq M$, then the probability that $\mathbb{X}$ has remnant is greater than $1-\varepsilon$.

Proof. Given $\varepsilon>0$, choose $\eta>0$ such that $(1-\eta)^{2}=1-\varepsilon$. Say that $\mathbb{X}>m$ if all $\left|X_{i}\right|>m$ for some integer $m$ and similarly, $\mathbb{X} \leq M$ if all $\left|X_{i}\right| \leq M$ for some integer $M$. Note that

$\operatorname{Prob}(\mathbb{X}$ has remnant $\mid \mathbb{X} \leq M) \geq \operatorname{Prob}(\mathbb{X}$ has remnant and $\mathbb{X}>m \mid \mathbb{X} \leq M)$ $=\operatorname{Prob}(\mathbb{X}$ has remnant $\mid M<\mathbb{X} \leq \mathbb{M}) \cdot \operatorname{Prob}(\mathbb{X}>m \mid \mathbb{X} \leq M)$.

We first show that we can choose $m$ so that

$$
\operatorname{Prob}(\mathbb{X} \text { has remnant } \mid m<\mathbb{X} \leq M)>1-\eta \text {. }
$$

Now

$\operatorname{Prob}(\mathbb{X}$ has no remnant $\mid m<\mathbb{X} \leq M) \leq n$,

$\operatorname{Prob}\left(X_{i}\right.$ has no remnant $\left.\mid m<\mathbb{X} \leq M\right)$. 
If $X_{i}$ has no remnant, then either $\left|P_{i}\right| \geq\left|X_{i}\right| / 2$ or $\left|S_{i}\right| \geq\left|X_{i}\right| / 2$. The probability that there exists $X_{j}^{\varepsilon_{j}}=U \bar{X}_{j}$ where $X_{i}^{\varepsilon_{i}}=U \bar{X}_{i},|U| \geq\left|X_{i}\right| / 2 \geq m / 2$ and $X_{j}^{\varepsilon_{j}} \neq$ $X_{i}^{\varepsilon_{i}}$ is less than or equal to $(2 n-1)(2 n)^{-m / 2}$, by independence. Therefore

$$
\operatorname{Prob}\left(X_{i} \text { has no remnant } \mid m<\mathbb{X} \leq M\right) \leq 2(2 n-1)(2 n)^{-m / 2}
$$

and so

$\operatorname{Prob}(\mathbb{X}$ has no remnant $\mid m<\mathbb{X} \leq M) \leq 2 n(2 n-1)(2 n)^{-m / 2}<(2 n)^{2-m / 2}$.

We can choose $m$ so that $(2 n)^{2-m / 2}<\eta$.

We will now show that given such an $m$ we can choose $M$ so that

$$
\operatorname{Prob}(\mathbb{X}>m \mid \mathbb{X} \leq M)>1-\eta \text {. }
$$

We use the fact that

$$
\operatorname{Prob}(\mathbb{X} \ngtr m \mid \mathbb{X} \leq M) \leq n \operatorname{Prob}\left(\left|X_{i}\right| \leq m \mid \mathbb{X} \leq M\right) .
$$

Let $W(k)$ denote the number of reduced words in $G$ of length $k$, then the number of reduced words of length less than or equal to $m$ is

$$
\begin{aligned}
& \sum_{0 \leq k \leq m} W(k)=1+\sum_{1 \leq k \leq m} 2 n(2 n-1)^{k-1} \\
& \quad=1+2 n\left(1-(2 n-1)^{m-1}\right) /(1-(2 n-1))=(2 n-1)^{m-1}
\end{aligned}
$$

and thus

$$
\operatorname{Prob}(\mathbb{X} \ngtr m \mid \mathbb{X} \leq M) \leq n(2 n-1)^{m-1} /(2 n-1)^{M-1}=n(2 n-1)^{m-M} .
$$

Thus, given $m$, we choose $M$ so that $n(2 n-1)^{m-M}<\eta$. Therefore

$$
\operatorname{Prob}(\mathbb{X} \text { has remnant } \mid \mathbb{X} \leq M)>(1-\eta)^{2}=1-\varepsilon
$$

Our main result in this section is to show that the conditions of Theorem 3.1 determine the fixed point classes of a given map $f$ if $\mathbb{X}$ has remnant. In other words,

Theorem 3.8. If $f: C \rightarrow C$ is a map such that $\mathbb{X}$ has remnant, then $f$ is $W$ characteristic.

The converse of Theorem 3.8 is not true.

Example 3.9. Suppose $\pi_{1}(C)$ is generated by two elements $a_{1}, a_{2}$ and $X_{1}=a_{1} a_{2}$ and $X_{2}=a_{2}$. Clearly $\mathbb{X}=\left\{X_{1}, X_{2}\right\}$ does not have remnant and cannot be simplified by (S1) and (S2) in Section 2. There are three fixed points and each fixed point is directly related to the other two. Therefore, there is one fixed point class and by definition, $f$ is $W$-characteristic.

We also need to state that not all $f$ are $W$-characteristic.

Example 3.10. Suppose $\pi_{1}(C)$ is generated by $a_{1}$ and $a_{2}$ and $f: C \rightarrow C$ is a map such that

$$
X_{1}=a_{1} a_{2} a_{1} a_{2} \quad \text { and } \quad X_{2}=a_{1}^{-1} .
$$

There are two fixed points and $x_{0}$, and if we number them in order of occurrence, then

$$
\begin{array}{ll}
W_{0}=1, & \bar{W}_{0}=1, \\
W_{1}=1, & \bar{W}_{1}=a_{2}^{-1} a_{1}^{-1} a_{2}^{-1}, \\
W_{2}=a_{1} a_{2}, & \bar{W}_{2}=a_{2}^{-1} .
\end{array}
$$


The only two fixed points which are directly related are $x_{0}$ and $x_{1}$. If $f$ were $W$ characteristic, then $x_{2}$ would not be in the same fixed point class as $x_{1}$ and $x_{0}$. However, if we let $z=a_{2}$, then

$$
W_{1}^{-1} f(z) W_{2}=a_{1}^{-1}\left(a_{1} a_{2}\right)=a_{2}=z .
$$

Therefore, $x_{1}$ and $x_{2}$ are in the same fixed point class by Lemma 1.5 and so $f$ is not $W$-characteristic.

We can find a map $f^{\prime}$ which has the same Nielsen number as $f$ and is $W$ characteristic. Define $\phi: C \rightarrow C$ by $\phi\left(a_{1}\right)=a_{2}^{-1} a_{1}$ and $\phi\left(a_{2}\right)=a_{2}$. Therefore, $\phi^{-1}\left(a_{1}\right)=a_{2} a_{1}$ and $\phi^{-1}\left(a_{2}\right)=a_{2}$ and we let $f^{\prime}=\phi \circ f \circ \phi^{-1}$. By commutativity of the Nielsen number, $N(f)=N\left(f^{\prime}\right)$. Now, $f^{\prime}\left(a_{1}\right)=a_{1} a_{2}$ and $f^{\prime}\left(a_{2}\right)=a_{1}^{-1} a_{2}$ and since $\left\{a_{1} a_{2}, a_{1}^{-1} a_{2}\right\}$ has remnant, then $f^{\prime}$ is $W$-characteristic.

Given any map $f: C \rightarrow C$, an interesting question would be: Under what conditions can we find a map $f^{\prime}: C \rightarrow C$ such that $N\left(f^{\prime}\right)=N(f)$ and $f^{\prime}$ is $W$-characteristic?

The rest of this section will be devoted to a proof of Theorem 3.8.

Remark 3.11. If $x_{1}$ and $x_{2}$ are in the same fixed point class, then by Lemma 1.5 there exists $z \in G$ such that $z=W_{1}^{-1} f(z) W_{2}$. Since Lemma 3.5 deals with the cancelation that occurs within $f(z)$, and since $W_{1}^{-1}$ and $W_{2}$ are reduced words, we need be concerned with only the cancelation occurring between $f(z)$ and each of the $W_{i}$. By Lemma 3.5, if $z=a_{i_{1}}^{\varepsilon_{1}} \cdots a_{i_{k}}^{\varepsilon_{k}}$, then $f(z)=U R_{1} \cdots R_{k} V^{-1}$ where $U=M\left(X_{i_{1}}^{\varepsilon_{1}}, \mathbb{X}\right)$ and $V=M\left(X_{i_{k}}^{-\varepsilon_{k}}, \mathbb{X}\right)$. Because of the symmetry involved, we will only consider $W_{1}^{-1}$. There are three possibilities:

(R1) If $W_{1}^{-1}$ does not cancel at all with $f(z)$, then $\left|W_{1}^{-1} f(z)\right|=\left|W_{1}^{-1}\right|+|f(z)|$.

(R2) Suppose $W_{1}^{-1}$ cancels at least partially with $U$ but not at all with $R_{1}$, then $\left|W_{1}^{-1} f(z)\right|=\left|W_{1}^{-1} U\right|+\left|R_{1} \cdots R_{k} V\right|$. If (R1) or (R2) holds, we say that $W_{1}$ cancels at most with an $\mathrm{MCF}$.

(R3) If the first two cases do not hold, then $W_{1}$ cancels at least partially with $R_{1}$. By Definition 3.3, $U$ is the longest portion of $X_{i_{1}}^{\varepsilon_{1}}$ that cancels in any product of the form $X_{j}^{-1} X_{i_{1}}^{\varepsilon_{1}}$ where $X_{j} \neq X_{i_{1}}^{\varepsilon_{1}}$ and by Lemma $1.3, X_{m}=W_{1} a_{m} \bar{W}_{1}$ for some $m$. Therefore, since more than $U$ is cancelled, $X_{m}=X_{i_{1}}^{\varepsilon_{1}}$ and the condition $z=W_{1}^{-1} f(z) W_{2}$ of Lemma 1.5 becomes

$$
\begin{aligned}
a_{i_{1}} z_{1} & =z=W_{1}^{-1} f\left(a_{i_{1}} z_{1}\right) W_{2}=W_{1}^{-1} X_{i_{1}} f\left(z_{1}\right) W_{2} \\
& =a_{i_{1}} \bar{W}_{1}^{-1} f\left(z_{1}\right) W_{2} .
\end{aligned}
$$

Thus

$$
z_{1}=\bar{W}_{1}^{-1} f\left(z_{1}\right) W_{2} .
$$

By the same argument, we conclude that $\bar{W}_{1}$ cancels at most with an MCF because if not, $f\left(z_{1}\right)$ must start with $X_{i_{1}}^{-1}$, which would imply that $z_{1}$ starts with $a_{i_{1}}^{-1}$. However, this would imply that $z=a_{i_{1}} a_{i_{1}}^{-1} z_{2}$, which is not reduced. We will refer to case (R3) by saying that $W_{1}$ cancels with a remnant.

Let $Z_{i}$ be a general reduced word.

Lemma 3.12. Suppose that $z=Z_{1}^{-1} f(z) Z_{2}$ where $Z_{1}, Z_{2}$ and $z \neq 1$ are reduced words. If $Z_{1}$ and $Z_{2}$ cancel at most with an $M C F$, then

(1) Each of $Z_{1}$ and $Z_{2}$ is either trivial or an $M C F$. 
(2) The reduced form of $Z_{1}^{-1} f(z) Z_{2}$ consists only of powers of remnants $\bar{X}_{i}$ or, equivalently, all the MCF's are cancelled completely.

(3) If $\Phi_{a_{i}}(z) \neq 0$, then $\bar{X}_{i}=a_{i}$.

Proof. We will let $A_{i}=\Phi_{a_{i}}(z)$, which counts the number of occurrences of $a_{i}$ and $a_{1}^{-1}$ in $z$. We can also suppose that $z=a_{i_{1}}^{\varepsilon_{1}} \cdots a_{i_{k}}^{\varepsilon_{k}}$ and $f(z)=U R_{1} \cdots R_{k} V^{-1}$, as in Lemma 3.5. There are three possibilities which satisfy the hypothesis. We verify conclusions (1) and (2) in each case.

(a) If neither of the $Z_{i}$ cancel at all with $f(z)$, then by (R1),

$$
\begin{aligned}
\sum_{1 \leq i \leq n} A_{i} & =|z|=\left|Z_{1}^{-1} f(z) Z_{2}\right| \\
& =\left|Z_{1}^{-1}\right|+\sum_{1 \leq i \leq n}\left(A_{i}\left|\bar{X}_{i}\right|\right)+E+\left|Z_{2}\right|
\end{aligned}
$$

where $E$ is the sum of the lengths of all the uncancelled portions of MCF's in $f(z)$. Since $\bar{X}_{i}$ is non-trivial for all $i$, then

(1) $\left|Z_{i}\right|=0$ which implies that $Z_{i}=1$ for $i=1,2$,

(2) $E=0$ and so all MCF's are completely cancelled in $f(z)$.

(b) Suppose that one of the $Z_{i}$ does not cancel at all and the other one cancels at least partially with an MCF. Because of the symmetry involved, we can suppose that $Z_{1}^{-1}$ cancels at least partially with $U$ and $Z_{2}$ does not cancel at all with $f(z)$. Then we have from (R2) that

$$
\begin{aligned}
\sum_{1 \leq i \leq n} A_{i} & =\left|Z_{1}^{-1} f(z) Z_{2}\right| \\
& =\left|Z_{1}^{-1} U\right|+\sum_{1 \leq i \leq k} A_{i}\left|\bar{X}_{i}\right|+(E-|U|)+\left|Z_{2}\right|
\end{aligned}
$$

and therefore

(1) $Z_{1}=U$ and $Z_{2}=1$,

(2) $E=|U|$ which implies that $U$ was the only MCF not cancelled completely in $f(z)$ and it is cancelled completely by $Z_{1}$.

(c) If $Z_{1}^{-1}$ cancels at least partially with $U$ and $Z_{2}$ cancels at least partially with $V^{-1}$, then (R2) implies that

$$
\begin{aligned}
\sum_{1 \leq i \leq n} A_{i} & =\left|Z_{1}^{-1} f(z) Z_{2}\right| \\
& =\left|Z_{1}^{-1} U\right|+\sum_{1 \leq i \leq n} A_{i}\left|\bar{X}_{i}\right|+(E-|U|-|V|)+\left|V^{-1} Z_{2}\right| .
\end{aligned}
$$

Therefore we have

(1) $Z_{1}=U$ and $Z_{2}=V$,

(2) $E=|U|+|V|$ which implies that all the other MCF's were cancelled completely in $f(z)$ and $U$ and $V$ are completely cancelled by the $Z_{i}$.

We have shown that conclusions (1) and (2) hold in all cases and it remains to verify (3). By (2), $Z_{1}^{-1} f(z) Z_{2}=\bar{X}_{i_{1}}^{\varepsilon_{1}} \cdots \bar{X}_{i_{k}}^{\varepsilon_{i}}$ and so $\left|Z_{1}^{-1} f(z) Z_{2}\right|=\sum_{1 \leq i \leq n} A_{i}\left|\bar{X}_{i}\right|$. Since $|z|=\sum_{i} A_{i}$, then $A_{i} \neq 0$ implies that $\left|\bar{X}_{i}\right|=1$. Write $z=a_{i_{1}}^{\varepsilon_{1}} z_{1}$, then $a_{i_{1}}^{\varepsilon_{1}} z_{1}=$ $\bar{X}_{i_{1}}^{\varepsilon_{1}} \cdots \bar{X}_{i_{k}}$ which, since $|\bar{X}|=1$, implies that $\bar{X}_{i_{1}}^{\varepsilon_{1}}=a_{i_{1}}^{\varepsilon_{1}}$ and thus $\bar{X}_{i_{1}}=a_{i_{1}}$. 
Proof of Theorem 3.8. If $x_{1}$ and $x_{2}$ are in the same fixed point class, then by Lemma 1.5, there exists a word $z$ such that $z=W_{1}^{-1} f(z) W_{2}$. We will show that $x_{1}$ and $x_{2}$ are related by a set of intermediate fixed points.

If $z=1$, then $W_{1}=W_{2}$ [1.1], so let us now assume that $z$ is non-trivial and distinguish cases depending on how $W_{1}$ and $W_{2}$ cancel with $f(z)$. In each case, we will find a $z^{\prime}=Z_{1}^{-1} f\left(z^{\prime}\right) Z_{2}$ which satisfies the hypothesis of Lemma 3.12.

If $W_{1}$ and $W_{2}$ cancel at most with an MCF, then we may apply Lemma 3.12 with $Z_{1}=W_{1}$ and $Z_{2}=W_{2}$ and $z^{\prime}=z$.

If $W_{1}$ cancels with a remnant and $W_{2}$ cancels with at most an $\mathrm{MCF}$, then by (R3), $z=a_{i_{1}} z^{\prime}$ and $z^{\prime}=\bar{W}_{1}^{-1} f\left(z^{\prime}\right) W_{2}$ where $\bar{W}_{1}$ cancels at most with an MCF. We can again apply Lemma 3.12 with $Z_{1}=\bar{W}_{1}$ and $Z_{2}=W_{2}$.

If both $W_{1}$ and $W_{2}$ cancels with more than an MCF, then we can do the same as above to get $z=a_{i_{1}} z^{\prime} a_{i_{k}}^{-1}$ where $z^{\prime}=\bar{W}_{1}^{-1} f\left(z^{\prime}\right) \bar{W}_{2}$. We can again apply Lemma 3.12 with $Z_{1}=\bar{W}_{1}$ and $Z_{2}=\bar{W}_{2}$.

Therefore we write $z^{\prime}=Z_{1}^{-1} f\left(z^{\prime}\right) Z_{2}$ where $Z_{i}=W_{i}$ or $\bar{W}_{i}$.

(1) If $z^{\prime}$ is trivial, then $Z_{1}=Z_{2}$ which corresponds to one of the conditions of Theorem 3.1.

(2) Suppose now that $z^{\prime}=a_{i_{1}}^{\varepsilon_{1}} \cdots a_{i_{k}}^{\varepsilon_{k}}$ is non-trivial. By Lemma 3.12(3), $X_{i_{1}}=$ $P_{i_{1}} \bar{X}_{i_{1}} S_{i_{1}}=P_{i_{1}} a_{i_{1}} S_{i_{1}}$. Let $x_{3}$ be the fixed point corresponding to the 0 of $a_{i_{1}}$ in $X_{i_{1}}$. If $\varepsilon_{1}=1$, that is $z^{\prime}=a_{i_{1}} z_{1}$, then $f\left(z^{\prime}\right)=X_{i_{1}} f\left(z_{1}\right)=P_{i_{1}} a_{i_{1}} S_{i_{1}} f\left(z_{1}\right)$ and we have

$$
z^{\prime}=Z_{1}^{-1} f(z) Z_{2}=Z_{1}^{-1} P_{i_{1}} a_{i_{1}} S_{i_{1}} f\left(z_{1}\right) Z_{2} .
$$

Now Lemma 3.12 states that all the $P_{j_{i}}$ and $S_{j_{i}}$ are cancelled completely so we conclude that $Z_{1}=P_{i_{1}}=W_{3}$. Similarly, if $\varepsilon_{1}=-1$, then $Z_{1}=S_{i_{1}}^{-1}=\bar{W}_{3}$. In either case, since $Z_{1}=W_{1}$ or $\bar{W}_{1}$, then some condition of Theorem 3.1 is satisfied and $x_{1}$ and $x_{3}$ are directly related. We can write $Z_{1}^{-1}=X_{i_{1}}^{\varepsilon_{1}}=$ $a_{i_{1}}^{\varepsilon_{1}} Z_{3}^{-1}$ where $Z_{3}$ is $S_{i_{1}}^{-1}=\bar{W}_{3}$ if $\varepsilon_{1}=1$ or $P_{i_{1}}=W_{3}$ if $\varepsilon_{1}=-1$. We now have that

$$
z^{\prime}=a_{i_{1}}^{\varepsilon_{1}} z_{1}=Z_{1}^{-1} X_{i_{1}}^{\varepsilon_{1}} f\left(z_{1}\right) Z_{2}=a_{i_{1}}^{\varepsilon_{1}} Z_{3}^{-1} f\left(z_{1}\right) Z_{2}
$$

which is equivalent to

$$
z_{1}=Z_{3}^{-1} f\left(z_{1}\right) Z_{2} \text {. }
$$

Since $\left(z_{1}\right)_{s} \neq a_{i_{1}}^{-\varepsilon_{1}}$ by the reasoning at the end of (R3), then $Z_{3}$ cancels at most with an MCF. We can now repeat the entire argument with $Z_{3}$ and $Z_{2}$.

Every time we repeat (2), we get another intermediate fixed point $x_{i}$. Since $z^{\prime}$ is of finite length, there must be an $m$ such that $z_{m}$ is trivial, which implies that $x_{m+2}$ and $x_{2}$ are directly related. This completes the proof of Theorem 3.8.

\section{The Algorithm}

Given a map $f: C \rightarrow C$ and the corresponding set of reduced words $\mathbb{X}=$ $\left\{X_{1}, \ldots, X_{n}\right\}$, the algorithm consists of the following steps:

(1) Perform all possible simplification steps (S1) and (S2) and determine if the resulting map is $W$-characteristic.

(2) If so, determine $W_{j}$ and $\bar{W}_{j}$ for each fixed point $x_{j}$.

(3) Determine the fixed point classes and calculate $N(f)$. 
We will present an example of the calculation of $N(f)$. Suppose $\pi_{1}(C)$ is generated by five elements and the set $\mathbb{X}=\left\{X_{1}, \ldots, X_{5}\right\}$ is given by

$$
\begin{aligned}
& X_{1}=a_{1}^{2} a_{2}^{-2} a_{5} a_{4}^{-1} a_{3}^{-1} a_{1} a_{3}^{-1} a_{2} a_{1}^{-1}, \\
& X_{2}=a_{1} a_{4}^{2} a_{2}^{2} a_{5}^{-1} a_{3}^{-1} a_{1}^{-2}, \\
& X_{3}=a_{1} a_{4} a_{3} a_{2} a_{1}^{-1}, \\
& X_{4}=a_{1}^{2} a_{3} a_{5} a_{2}^{-2} a_{4}^{-2} a_{1} a_{3} a_{5} a_{2}^{-2} a_{4}^{-2}, a_{1}^{-1}, \\
& X_{5}=1 .
\end{aligned}
$$

Step 1: Apply (S1) and (S2) and check if the resulting map is $W$-characteristic (a) Perform all possible (S1) and (S2).

The simplifications (S1) and (S2) are defined in Section 2. Notice in our example that each $X_{i}=a_{1} X_{i}^{\prime} a_{1}^{-1}$ or $X_{i}=1$, and so we can perform the simplification (S1) to get a new set

$$
\begin{aligned}
& X_{1}=a_{1} a_{2}^{-2} a_{5} a_{4}^{-1} a_{3}^{-1} a_{1} a_{3}^{-1} a_{2}, \\
& X_{2}=a_{4}^{2} a_{2}^{2} a_{5}^{-1} a_{3}^{-1} a_{1}^{-1}, \\
& X_{3}=a_{4} a_{3} a_{2}, \\
& X_{4}=a_{1} a_{3} a_{5} a_{2}^{-2} a_{4}^{-2} a_{1} a_{3} a_{5} a_{2}^{-2} a_{4}^{-2}, \\
& X_{5}=1 .
\end{aligned}
$$

Notice now that $X_{2}, X_{4}$ and $X_{5}$ are multiples of the subword $a_{4}^{2} a_{2}^{2} a_{5}^{-1} a_{3}^{-1} a_{1}^{-1}$. Renumber the $a_{i}$ by interchanging $a_{2}$ and $a_{3}$ and thus the corresponding $X_{2}$ and $X_{3}$ to get

$$
\begin{aligned}
& X_{1}=a_{1} a_{3}^{-2} a_{5} a_{4}^{-1} a_{2}^{-1} a_{1} a_{2}^{-1} a_{3}, \\
& X_{2}=a_{4} a_{2} a_{3}, \\
& X_{3}=U, \\
& X_{4}=U^{-2}, \\
& X_{5}=U^{0},
\end{aligned}
$$

where $U=a_{4}^{2} a_{3}^{2} a_{5}^{-1} a_{2}^{-1} a_{1}^{-1}$. We can therefore perform the simplification (S2) with

$$
g_{\#}\left(a_{i}\right)= \begin{cases}b_{i} & \text { if } i=1,2,3 \\ b_{3}^{-2} & \text { if } i=4 \\ 1 & \text { if } i=5\end{cases}
$$

and

$$
h_{\#}\left(b_{i}\right)= \begin{cases}X_{i} & \text { if } i=1,2 \\ U & \text { if } i=3\end{cases}
$$

and look at the set

$$
\begin{aligned}
& g_{\#} h_{\#}\left(b_{1}\right)=b_{1} b_{3}^{-2} b_{3}^{2} b_{2}^{-1} b_{1} b_{2}^{-1} b_{3}=b_{1} b_{2}^{-1} b_{1} b_{2}^{-1} b_{3}, \\
& g_{\#} h_{\#}\left(b_{2}\right)=b_{3}^{-2} b_{2} b_{3} \\
& g_{\#} h_{\#}\left(b_{3}\right)=b_{3}^{-4} b_{3}^{2} b_{2}^{-1} b_{1}^{-1}=b_{3}^{-2} b_{2}^{-1} b_{1}^{-1} .
\end{aligned}
$$


We will set $a_{i}=b_{i}$ and $X_{i}=g_{\#} h_{\#}\left(b_{i}\right)$ to get

$$
\begin{aligned}
& X_{1}=a_{1} a_{2}^{-1} a_{1} a_{2}^{-1} a_{3}, \\
& X_{2}=a_{3}^{-2} a_{2} a_{3}, \\
& X_{3}=a_{3}^{-2} a_{2}^{-1} a_{1}^{-1} .
\end{aligned}
$$

We note that we can no longer do either (S1) or (S2) and let $f^{\prime}$ be the map corresponding to $\mathbb{X}^{\prime}=\left\{X_{1}, X_{2}, X_{3}\right\}$. If at this point, we only had one $X_{i}$, then we would apply Theorem 2.1 .

(b) Verify that $f^{\prime}$ is $W$-characteristic.

We will in fact show that $\mathbb{X}^{\prime}$ has remnant. The reader can check that all MCF's are contained in the brackets. See Example 3.4 for more detail on finding MCF's.

$$
\begin{aligned}
& X_{1}=\left[a_{1}\right] a_{2}^{-1} a_{1} a_{2}^{-1}\left[a_{3}\right], \\
& X_{2}=\left[a_{3}^{-2}\right] a_{2}\left[a_{3}\right], \\
& X_{3}=\left[a_{3}^{-2}\right] a_{2}^{-1}\left[a_{1}^{-1}\right] .
\end{aligned}
$$

Since each $X_{i}$ has a portion which is not contained in any MCF, then $X=$ $\mathbb{X}\left\{X_{1}, X_{2}, X_{3}\right\}$ has remnant and therefore $f^{\prime}$ is $W$-characteristic by Theorem 3.8.

Step 2: Determine the $W_{j}$ and $\bar{W}_{j}$.

$$
\begin{aligned}
& X_{1}=\mathbf{a}_{1} a_{2}^{-1} \mathbf{a}_{1} a_{2}^{-1} a_{3}, \\
& X_{2}=a_{3}^{-2} \mathbf{a}_{2} a_{3}, \\
& X_{3}=\mathbf{a}_{3}^{-1} \mathbf{a}_{3}^{-1} a_{2}^{-1} a_{1}^{-1} .
\end{aligned}
$$

The letters in boldface, where $a_{i}$ appears in $X_{i}$, indicate the occurrence of a fixed point. We will number these fixed points $x_{1}, \ldots, x_{5}$ in order of occurrence. We therefore have six fixed points, including $x_{0}$, and we will determine the corresponding $W_{i}$ and $\bar{W}_{i}$. See Lemma 1.2 and Example 1.4 to recall the definition of $W_{i}$ and $\bar{W}_{i}$.

$$
\begin{array}{ll}
W_{0}=1, & \bar{W}_{0}=1, \\
W_{1}=1, & \bar{W}_{1}=a_{3}^{-1} a_{2} a_{1}^{-1} a_{2}, \\
W_{2}=a_{1} a_{2}^{-1}, & \bar{W}_{2}=a_{3}^{-1} a_{2}, \\
W_{3}=a_{3}^{-2}, & \bar{W}_{3}=a_{3}^{-1}, \\
W_{4}=a_{3}^{-1}, & \bar{W}_{4}=a_{1} a_{2} a_{3}^{2}, \\
W_{5}=a_{3}^{-2}, & \bar{W}_{5}=a_{1} a_{2} a_{3} .
\end{array}
$$

Step 3: Determine the fixed point classes and calculate $N\left(f^{\prime}\right)=N(f)$.

By comparing the $W_{i}$ and $\bar{W}_{i}$, we see that the following pairs of fixed points are directly related: $\left(x_{0}, x_{1}\right),\left(x_{3}, x_{4}\right),\left(x_{3}, x_{5}\right)$. By Theorem 3.8 , we have the following fixed point classes: $F_{1}=\left\{x_{0}, x_{1}\right\}, F_{2}=\left\{x_{2}\right\}$ and $F_{3}=\left\{x_{3}, x_{4}, x_{5}\right\}$. We calculate that $i\left(F_{1}\right)=1-1=0, i\left(F_{2}\right)=-1$ and $i\left(F_{3}\right)=-1+1+1=1$ (see the comment before Example 1.4). Therefore, $N(f)=2$.

\section{The JiAng-Guo IneQuality}

B. Jiang and G. Guo [JG] proved that the inequality $|L(f)-\chi(M)| \leq N(f)-$ $\chi(M)$ holds for surface homeomorphisms $f: M \rightarrow M$ and asked if it is true for all maps of surfaces. We will show that it is true if the induced map on the fundamental 
group is homotopic to a $W$-characteristic map and therefore, by Theorems 3.7 and 3.8 , it is true for most maps.

Theorem 5.1. If $f: C \rightarrow C$ is $W$-characteristic, then

$$
|L(f)-\chi(C)| \leq N(f)-\chi(C)
$$

Since the Lefschetz number, the Euler characteristic and the Nielsen number are homotopy and homotopy-type invariant, we have

Corollary 5.2. If $F: M \rightarrow M$ is a self-map of a hyperbolic surface with boundary and the induced map of the fundamental group can be viewed as a map $f: C \rightarrow C$ which is homotopic to a map which is $W$-characteristic, then

$$
|L(F)-\chi(M)| \leq N(F)-\chi(M)
$$

For convenience, if a fixed point $x_{i}$ has positive index, we will call it a positive fixed point and if a fixed point class has positive index, we will call it a positive class. Similarly, for negative index. Furthermore, we will always assume that $\varepsilon_{i} \in\{-1,1\}$ and that the fixed point $x_{j}$ lies on $C_{i_{j}}$. If $F_{i}$ is a fixed point class, then $\mathbb{A}^{+}(i)$ will denote the number of positive fixed points in $F_{i}$. Let $\mathbb{A}_{0}^{+}, \mathbb{A}_{p}^{+}, \mathbb{A}_{n}^{+}$, and $\mathbb{A}^{+}$be the total number of positive fixed points in the inessential fixed point classes, the positive fixed point classes, the negative fixed point classes, and all the classes, respectively. For the number of negative fixed points, we use $\mathbb{A}_{0}^{-}, \mathbb{A}_{p}^{-}, \mathbb{A}_{n}^{-}$and $\mathbb{A}^{-}$. Suppose there are $c^{+}$positive classes and $c^{-}$negative classes.

If $\pi_{1}(C)$ has $n$-generators, then $\chi(C)=1-n$. By definition, $L(f)=\mathbb{A}^{+}-\mathbb{A}^{-}$ and $N(f)=c^{+}+c^{-}$. We will look more carefully at the relationship between $\mathbb{A}^{+}(i)$ and $\mathbb{A}^{-}(i)$ for each type of class.

Since inessential fixed point classes are defined to have an index of zero, then $\mathbb{A}_{0}^{+}=\mathbb{A}_{0}^{-}$.

In a positive class, $\mathbb{A}^{+}(i)>\mathbb{A}^{-}(i)$ and, in particular,

Lemma 5.3. If $f$ is $W$-characteristic and $F_{i}$ is a positive fixed point class, then $\mathbb{A}^{+}(i)=\mathbb{A}^{-}(i)+1$. Therefore, $\mathbb{A}_{p}^{+}=\mathbb{A}_{n}^{-}+c^{+}$.

We will postpone the proof until later.

If $F_{i}$ is a negative fixed point class, then we can write $\mathbb{A}^{-}(i)=\mathbb{A}^{+}(i)+j_{i}$ where $j_{i}>0$. If we let $J$ be the sum of the $j_{i}$ over all negative classes, then $J=\sum_{i}\left(\mathbb{A}^{-}(i)-\mathbb{A}^{+}(i)\right)=\mathbb{A}_{n}^{-}-\mathbb{A}_{n}^{+}$. We will prove

Lemma 5.4. If $f$ is $W$-characteristic, then $J \leq 2 n+c^{-}-2$.

We will suppose Lemmas 5.3 and 5.4 hold and prove Theorem 5.1.

Proof of Theorem 5.1. The inequality $|L(f)-\chi(M)| \leq N(f)-\chi(M)$ is equivalent to showing that $N(f) \geq L(f)$ and $N(f)+L(f)-2 \chi(M) \geq 0$.

By Lemma 5.3,

$$
L(f)=\mathbb{A}^{+}-\mathbb{A}^{-}=\left(\mathbb{A}_{0}^{+}-\mathbb{A}_{0}^{-}\right)+\left(\mathbb{A}_{p}^{+}-\mathbb{A}_{p}^{-}\right)+\left(\mathbb{A}_{n}^{+}-\mathbb{A}_{n}^{-}\right)=c^{+}-J .
$$

Since $J \geq 0$, then $L(f) \leq c^{+} \leq N(f)$.

By Lemma 5.4, we have

$$
\begin{aligned}
N(f) & +L(f)-2 \chi(C)=\left(c^{+}+c^{-}\right)+\left(c^{+}-J\right)-2(1-n) \\
& =2 c^{+}+\left(c^{-}+2 n-2\right)-J \\
& \geq 2 c^{+}+\left(c^{-}+2 n-2\right)-\left(2 n+c^{-}-2\right)=2 c^{+} \geq 0 .
\end{aligned}
$$


From Theorems 3.8 and 5.1 we have the following result:

Corollary 5.5. If $f: C \rightarrow C$ is a map such that $\mathbb{X}$ has remnant, then

$$
|L(f)-\chi(C)| \leq N(f)-\chi(C) .
$$

The following is a corollary to Lemmas 5.3 and 5.4 .

Theorem 5.6. If $f$ is a $W$-characteristic and $F_{i}$ is a fixed point class of $f$ and $i\left(F_{i}\right)$ is the index of $F_{i}$, then $1-2 n \leq i\left(F_{i}\right) \leq 1$.

Proof. By Lemma 5.3, we know that $i\left(F_{i}\right) \leq 1$. If $F_{1}, \ldots, F_{c^{-}}$are the negative fixed point classes, then $i\left(F_{i}\right)=\mathbb{A}^{+}(i)-\mathbb{A}^{-}(i)=-j_{i}$. Since

$$
J=\sum_{1 \leq i \leq c^{-}} j_{i} \leq 2 n+c^{-}-2
$$

by Lemma 5.4 , then $j_{i} \leq 2 n+c^{-}-2-\left(c^{-}-1\right)=2 n-1$.

We will devote the remainder of the section to proving Lemmas 5.3 and 5.4.

Lemma 5.7. If $f$ is $W$-characteristic, then no two positive fixed points can be directly related.

Proof. If $x_{1}$ and $x_{2}$ are positive fixed points, neither of which is $x_{0}$, then for some $i_{1}$ and $i_{2}$, we can write $X_{i_{1}}^{\varepsilon_{1}}=Z_{1} a_{i_{1}}^{-\varepsilon_{1}} \ldots$ and $X_{i_{2}}^{\varepsilon_{2}}=Z_{2} a_{i_{2}}^{-\varepsilon_{2}} \ldots$ where

$$
Z_{k} a_{i_{k}}^{-\varepsilon_{k}}= \begin{cases}W_{k} & \text { if } \varepsilon_{k}=1, \\ \bar{W}_{k} & \text { if } \varepsilon_{k}=-1\end{cases}
$$

Therefore, for the conditions of Theorem 3.1 to hold, there exists $\varepsilon_{1}$ and $\varepsilon_{2}$ so that $Z_{1} a_{i_{1}}^{-\varepsilon_{1}}=Z_{2} a_{i_{2}}^{-\varepsilon_{2}}$. This implies that $i_{1}=i_{2}, \varepsilon_{1}=\varepsilon_{2}$ and $Z_{1}=Z_{2}$ which happens only when $x_{1}=x_{2}$.

If $x_{1}=x_{0}$, then $W_{1}=\bar{W}_{1}=1$. However, neither $W_{2}$ nor $\bar{W}_{2}$ can be trivial, by Lemma 1.2 , and therefore $x_{1}$ and $x_{2}$ cannot be directly related.

Define

$$
T\left(U_{i}\right)=\left\{x_{j} \in \operatorname{Fix}(f) \mid W_{j}=U_{i} \quad \text { or } \quad \bar{W}_{j}=U_{i}\right\} .
$$

Clearly a point in $T\left(U_{i}\right)$ is directly related to every other point in $T\left(U_{i}\right)$ and therefore each $T\left(U_{i}\right)$ is contained in a fixed point class.

Proof of Lemma 5.3. Since $F_{i}$ is positive, then $\mathbb{A}^{+}(i)-\mathbb{A}^{-}(i) \geq 1$. If $F_{i}=\left\{x_{j}\right\}$, then $i\left(F_{i}\right)=1$. Otherwise, since $f$ is $W$-characteristic, we can write $F_{i}=T\left(U_{1}\right) \cup$ $\cdots \cup T\left(U_{m_{i}}\right)$. If $T\left(U_{j}\right)$ did not intersect any of the other $T\left(U_{k}\right)$ in $F_{i}$, then none of the fixed points in $T\left(U_{j}\right)$ would be directly related to any of the other fixed points in $F_{i}$ and therefore $F_{i}=U\left(U_{j}\right)$. We conclude, then, that there must be at least $m_{i}-1$ points of $F_{i}$ that are in two $T\left(U_{j}\right)$. By Lemma 5.7, each $T\left(U_{i}\right)$ contains at most one positive fixed point and so if $r$ of the positive fixed points are contained in two of the $T\left(U_{i}\right)$, then $\mathbb{A}^{+}(i) \leq m_{i}-r$. Since there need to be at least $m_{i}-1$ points of $F_{i}$ in two $T\left(U_{i}\right)$, then $\mathbb{A}^{-}(i) \geq m_{i}-1-r$ and therefore, $\mathbb{A}^{+}(i)-\mathbb{A}^{-}(i) \leq m_{i}-r-\left(m_{i}-1-r\right)=1$.

Let $T^{-}\left(U_{i}\right)$ be the set of all the negative fixed points in $T\left(U_{i}\right)$ and let $\mathbb{S}=$ $\left\{U_{1}, \ldots, U_{M}\right\}$ where $\left|T^{-}\left(U_{i}\right)\right| \geq 1$ and $T\left(U_{i}\right)$ is contained in a negative fixed point class. Define $P\left(U_{i}\right)=\left\{\left(j, \varepsilon_{j}\right) \mid X_{j}^{\varepsilon_{j}}=U_{i} a_{j}^{\varepsilon} \cdots\right\}$. In other words, each element of 
$P\left(U_{i}\right)$ corresponds to an occurrence of $U_{i}$ which is followed by a negative fixed point.

To prove Lemma 5.4, we will make a series of claims.

Claim 5.8. If $V, Z \in \mathbb{S}, V \neq Z$, then $|P(V) \cap P(Z)| \leq 1$.

Proof. If $\left(i, \varepsilon_{i}\right)$ and $\left(j, \varepsilon_{j}\right)$ are distinct elements of $P(V) \cap P(Z)$, then

$$
X_{i}^{\varepsilon_{i}}=V a_{i}^{\varepsilon_{i}} \cdots=Z a_{i}^{\varepsilon_{i}} \cdots
$$

and

$$
X_{j}^{\varepsilon_{j}}=V a_{j}^{\varepsilon_{j}} \cdots=Z a_{j}^{\varepsilon_{j}} \cdots .
$$

Either both $V$ and $Z$ are trivial or $V$ and $Z$ are both maximal common factors for $X_{i}^{\varepsilon_{i}}$ and $X_{j}^{\varepsilon_{j}}$. However, this implies that $V=Z$ which contradicts the hypothesis.

Claim 5.9. Given $P\left(U_{1}\right), \ldots, P\left(U_{M}\right)$, we can find sets $\mathbb{S}_{1}, \ldots, \mathbb{S}_{N}$ such that for each $1 \leq \alpha \leq N, \mathbb{S}_{\alpha}=P\left(U_{\alpha_{1}}\right) \cup \cdots \cup P\left(U_{\alpha_{m_{\alpha}}}\right)$ and

(1) the $\mathbb{S}_{\alpha}$ are disjoint,

(2) $\bigcup_{1 \leq \alpha \leq N} \mathbb{S}_{\alpha}=\bigcup_{1 \leq j \leq M} P\left(U_{i}\right)$,

(3) given any $P\left(U_{\alpha_{k}}\right) \subset \mathbb{S}_{\alpha}$, we can order $\mathbb{S}_{\alpha}=P\left(U_{\sigma\left(\alpha_{1}\right)}\right) \cup \cdots \cup P\left(U_{\sigma\left(\alpha_{m_{\alpha}}\right)}\right)$ where $\sigma\left(\alpha_{1}\right)=\alpha_{k}$ so that

$$
\left|P\left(U_{\sigma\left(\alpha_{j}\right)}\right) \cap\left[P\left(U_{\sigma\left(\alpha_{1}\right)}\right) \cup \cdots \cup P\left(U_{\sigma\left(\alpha_{j-1}\right)}\right)\right]\right| \geq 1
$$

for all $1 \leq j \leq m_{\alpha}$.

Proof. We will do induction on $M$, the number of $P\left(U_{i}\right)$. If $M=1$ and we set $\mathbb{S}_{1}=P\left(U_{1}\right)$, then $\mathbb{S}_{1}$ clearly satisfies (1)-(3).

Suppose that the claim is true for $M-1$, that is, we have found sets $\mathbb{S}_{1}, \ldots, \mathbb{S}_{N}$ which contain $P\left(U_{1}\right), \ldots, P\left(U_{M-1}\right)$ and satisfy (1)-(3).

Now consider $P\left(U_{M}\right)$. If $P\left(U_{M}\right)$ does not intersect any of the $\mathbb{S}_{\alpha}$, let $\mathbb{S}_{N+1}=$ $P\left(U_{M}\right)$. The sets $\mathbb{S}_{1}, \ldots, \mathbb{S}_{N+1}$ are clearly disjoint and their union is the union of the $P\left(U_{i}\right)$. By the induction hypothesis, (3) is satisfied by $\mathbb{S}_{1}, \ldots, \mathbb{S}_{N}$ and it is clearly satisfied by $\mathbb{S}_{N+1}$ as well.

Suppose now that $P\left(U_{M}\right)$ does intersect some of the $\mathbb{S}_{\alpha}$. We can assume that the $\mathbb{S}_{\alpha}$ have been numbered so that $\mathbb{S}_{K+1}, \ldots, \mathbb{S}_{N}$ are the only $\mathbb{S}_{\alpha}$ which intersect $P\left(U_{M}\right)$. We define $\mathbb{T}=P\left(U_{M}\right) \cup \mathbb{S}_{K+1} \cup \mathbb{S}_{K+2} \cup \cdots \cup \mathbb{S}_{N}$ and we will show that $\mathbb{S}_{1}, \ldots, \mathbb{S}_{K}, \mathbb{T}$ satisfy $(1)-(3)$. Since the $\mathbb{S}_{1}, \ldots, \mathbb{S}_{N}$ are disjoint and $\mathbb{S}_{1}, \ldots, \mathbb{S}_{K}$ do not intersect $P\left(U_{M}\right)$, then (1) is satisfied. Furthermore, since (2) holds for $\mathbb{S}_{1}, \ldots, \mathbb{S}_{N}$, it clearly holds for $\mathbb{S}_{1}, \ldots, \mathbb{S}_{K}, \mathbb{T}$ also.

By the induction hypothesis, (3) holds for $\mathbb{S}_{1}, \ldots, \mathbb{S}_{K}$ and so we only need to show that (3) holds for $\mathbb{T}$. Given any $P\left(U_{k}\right) \subset \mathbb{T}$ we wish to show that we can order $\mathbb{T}$ so that it starts with $P\left(U_{k}\right)$ and satisfies (*). Suppose that $P\left(U_{k}\right)=P\left(U_{M}\right)$. Since $\mathbb{S}_{\beta}$ intersects $P\left(U_{M}\right)$ for each $K+1 \leq \beta \leq N$, there is some $P\left(U_{i}\right) \subset \mathbb{S}_{\beta}$ which intersects $P\left(U_{M}\right)$. Since $\mathbb{S}_{\beta}$ satisfies (3) by the induction hypothesis, we order $\mathbb{S}_{\beta}$ to start with $P\left(U_{i}\right)$ and to satisfy $(*)$. Now $\mathbb{T}=P\left(U_{M}\right) \cup \mathbb{S}_{K+1} \cup \cdots \cup \mathbb{S}_{N}=P\left(U_{\alpha_{1}}\right) \cup \cdots \cup P\left(U_{\alpha_{m}}\right)$. Given any $P\left(U_{\alpha_{j}}\right) \subset \mathbb{T}$ for $j \geq 2$, we have that $P\left(U_{\alpha_{j}}\right) \subset \mathbb{S}_{\beta}$ for some $\beta$. Either $P\left(U_{\alpha_{i}}\right)$ is the initial term in the ordering of $\mathbb{S}_{\beta}$ and therefore intersects $P\left(U_{M}\right)$, or it intersects an earlier term in the ordering of $\mathbb{S}_{\beta}$. In either case, $(*)$ is satisfied.

Suppose now that $P\left(U_{k}\right) \neq P\left(U_{M}\right)$. Therefore, it must be contained in some $\mathbb{S}_{\gamma}$ which, by (3) and the induction hypothesis, we may order to start with $P\left(U_{k}\right)$ 
and to satisfy $(*)$. We will order all the other $\mathbb{S}_{\beta}$ to start with an element that intersects $P\left(U_{M}\right)$ and to satisfy $(*)$. Now write

$$
\mathbb{T}=\mathbb{S}_{\gamma} \cup P\left(U_{M}\right) \cup \bigcup_{\beta \neq \gamma} \mathbb{S}_{\beta}=P\left(U_{\alpha_{1}}\right) \cup \cdots \cup P\left(U_{\alpha_{m}}\right) .
$$

Consider any $P\left(U_{\alpha_{i}}\right) \subset \mathbb{T}$. Suppose $P\left(U_{\alpha_{i}}\right) \subset \mathbb{S}_{\beta}$ for some $\beta$. Either $P\left(U_{\alpha_{i}}\right)$ is the initial term in the ordering of $\mathbb{S}_{\beta}$ and intersects $P\left(U_{M}\right)$ or it intersects an earlier term in the ordering of $\mathbb{S}_{\beta}$. If $P\left(U_{\alpha_{i}}\right) \subset \mathbb{S}_{\gamma}$, then it will intersect an earlier term in $\mathbb{S}_{\gamma}$. If $P\left(U_{\alpha_{i}}\right)=P\left(U_{M}\right)$, then $P\left(U_{\alpha_{i}}\right)$ intersects some element in $\mathbb{S}_{\gamma}$ since $P\left(U_{M}\right)$ intersects $\mathbb{S}_{\gamma}$. Therefore $(*)$ holds and $\mathbb{T}$ satisfies (3).

Claim 5.10. If $\mathbb{S}_{\alpha}=P\left(U_{\alpha_{1}}\right) \cup \cdots \cup P\left(U_{\alpha_{m}}\right)$ satisfies $(*)$ and $U_{\alpha_{k}}$ has the smallest length among all $U_{\alpha_{i}}$, that is, $\left|U_{\alpha_{k}}\right| \leq\left|U_{\alpha_{i}}\right|$ for all $i$, then $U_{\alpha_{i}}=U_{\alpha_{k}} \cdots$.

Proof. We will do induction on $m$, the number of $P\left(U_{\alpha_{i}}\right)$ in $\mathbb{S}_{\alpha}$. If $m=2$ so $\mathbb{S}_{\alpha}=P\left(U_{\alpha_{1}}\right) \cup P\left(U_{\alpha_{2}}\right)$, then

$$
X_{i}^{\varepsilon_{i}}=U_{\alpha_{1}} a_{i}^{\varepsilon_{i}} \cdots=U_{\alpha_{2}} a_{i}^{\varepsilon_{i}} \cdots
$$

for some $\left(i, \varepsilon_{i}\right)$. If we assume $\left|U_{\alpha_{1}}\right| \leq\left|U_{\alpha_{2}}\right|$, then $U_{\alpha_{2}}=U_{\alpha_{1}} \cdots$.

Suppose the claim is true for $m-1$. Since $P\left(U_{\alpha_{1}}\right) \cup \cdots \cup P\left(U_{\alpha_{m-1}}\right)$ also satisfies $(*)$, then for some $k \leq m-1, U_{\alpha_{i}}=U_{\alpha_{k}} \cdots$ for all $1 \leq i \leq m-1$. Since $\mathbb{S}_{\alpha}$ satisfies $(*)$, then there exists at least one $j<m$ such that $\left|P\left(U_{\alpha_{m}}\right) \cap P\left(U_{\alpha_{j}}\right)\right|=1$. Therefore,

$$
X_{i}^{\varepsilon_{i}}=U_{\alpha_{m}} a_{i}^{\varepsilon_{i}} \cdots=U_{\alpha_{j}} a_{i}^{\varepsilon_{i}} \cdots
$$

for $\left(i, \varepsilon_{i}\right)=P\left(U_{\alpha_{m}}\right) \cap P\left(U_{\alpha_{j}}\right)$. If $\left|U_{\alpha_{m}}\right| \geq\left|U_{\alpha_{j}}\right|$, then by the induction hypothesis, $U_{\alpha_{m}}=U_{\alpha_{j}} \cdots=U_{\alpha_{k}} \cdots$ and the claim holds.

If $\left|U_{\alpha_{m}}\right| \leq\left|U_{\alpha_{j}}\right|$, then $U_{\alpha_{j}}=U_{\alpha_{m}} \cdots$, but we also have $U_{\alpha_{j}}=U_{\alpha_{k}} \cdots$ by the induction hypothesis, so $U_{\alpha_{m}} \cdots=U_{\alpha_{k}} \cdots$. If $\left|U_{\alpha_{m}}\right| \geq\left|U_{\alpha_{k}}\right|$, then we are done. If not, $U_{\alpha_{k}}=U_{\alpha_{m}} \cdots$ and by the induction hypothesis, $U_{\alpha_{i}}=U_{\alpha_{m}} \cdots$ for all $i$.

Claim 5.11. If $K$ is the total number of positive fixed points which are contained in a negative class and are contained in only one $T\left(U_{i}\right)$, then

$$
\sum_{1 \leq i \leq M}\left|P\left(U_{i}\right)\right| \geq J+M-c^{-}+K .
$$

Proof. By definition, each element in $T^{-}\left(U_{i}\right)$ contributes exactly once to the set $P\left(U_{i}\right)$. Therefore $\sum_{1 \leq i \leq M}\left|P\left(U_{i}\right)\right|=\sum_{1 \leq i \leq M}\left|T^{-}\left(U_{i}\right)\right|$. Furthermore, since all negative fixed points contribute to either one or two of the $T^{-}\left(U_{i}\right)$, then

$$
\mathbb{A}_{n}^{-}=\left|\bigcup_{i} T^{-}\left(U_{i}\right)\right|=\sum_{i}\left|T^{-}\left(U_{i}\right)\right|-\sum_{1 \leq j<k \leq M}\left|T^{-}\left(U_{j}\right) \cap T^{-}\left(U_{k}\right)\right|
$$

and therefore

$$
\sum_{1 \leq i \leq M}\left|P\left(U_{i}\right)\right|=\mathbb{A}_{n}^{-}+\sum_{1 \leq j<k \leq M}\left|T^{-}\left(U_{j}\right) \cap T^{-}\left(U_{k}\right)\right| .
$$

We now wish to find a lower bound for $\sum_{1 \leq j<k \leq M}\left|T^{-}\left(U_{j}\right) \cap T^{-}\left(U_{k}\right)\right|$. As we observed in the proof of Lemma 5.3, if we write $F_{i}=T\left(U_{1}\right) \cup \cdots \cup T\left(U_{m_{i}}\right)$, then 
$\sum_{1 \leq j<k \leq m_{i}}\left|T\left(U_{j}\right) \cap T\left(U_{k}\right)\right| \geq m_{i}-1$. Suppose that there are $K_{i}$ positive fixed points in $F_{i}$, each of which belongs to only one $T\left(U_{j}\right)$, then at most $\mathbb{A}^{+}(i)-K_{i}$ positive fixed points could account for the intersections $T\left(U_{j}\right) \cap T\left(U_{k}\right)$. Therefore, at least $m_{i}-1-\left(\mathbb{A}^{+}(i)-K_{i}\right)$ negative points must be in the intersections and so

$$
\begin{aligned}
\sum_{1 \leq j<k \leq M}\left|T^{-}\left(U_{j}\right) \cap T^{-}\left(U_{k}\right)\right| & \geq \sum_{i}\left(m_{i}-1-\left(\mathbb{A}^{+}(i)-K_{i}\right)\right) \\
& =M-c^{-}-\mathbb{A}_{n}^{+}+K
\end{aligned}
$$

where we are only summing over the negative $F_{i}$. Therefore,

$$
\sum_{1 \leq i \leq M}\left|P\left(U_{i}\right)\right| \geq \mathbb{A}_{n}^{-}+M-c^{-}-\mathbb{A}_{n}^{+}+K=J+M-c^{-}+K .
$$

Claim 5.12. If $\mathbb{S}_{\alpha}=P\left(U_{\alpha_{1}}\right) \cup \cdots \cup P\left(U_{\alpha_{m_{\alpha}}}\right)$ satisfies $(*)$, then

$$
\left|\mathbb{S}_{\alpha}\right| \geq \sum_{1 \leq i \leq m}\left|P\left(U_{\alpha_{i}}\right)\right|-\left(m_{\alpha}-1\right)
$$

Proof. Given $\mathbb{S}_{\alpha}$ ordered as above, let $\Gamma$ be the undirected graph whose vertices are the $P\left(U_{\alpha_{i}}\right)$ and which has an edge connecting $P\left(U_{\alpha_{i}}\right)$ and $P\left(U_{\alpha_{j}}\right)$ if and only if

$$
\begin{gathered}
P\left(U_{\alpha_{i}}\right) \cap P\left(U_{\alpha_{j}}\right)=\varnothing \text { and there is no }(k, l) \neq(i, j) \text { where } \\
k \geq i l \geq j \text { and } P\left(U_{\alpha_{i}}\right) \cap P\left(U_{\alpha_{j}}\right)=P\left(U_{\alpha_{k}}\right) \cap P\left(U_{\alpha_{l}}\right) .
\end{gathered}
$$

We claim that $\Gamma$ contains no cycles. Suppose $\Gamma$ contains a cycle which has vertices $P\left(U_{\beta_{1}}\right), \ldots, P\left(U_{\beta_{k}}\right)$ and edges $e_{(1,2)}, e_{(2,3)}, \ldots, e_{(k-1, k)}, e_{(k, 1)}$ where $e_{(i, j)}$ connects $P\left(U_{\beta_{i}}\right)$ and $P\left(U_{\beta_{j}}\right)$ and $k \geq 3$. Because each edge represents an intersection, $\mathbb{T}=P\left(U_{\beta_{1}}\right) \cup \cdots \cup P\left(U_{\beta_{k}}\right)$ satisfies $(*)$ and so by Claim 5.10, there is some $r$ such that $U_{\beta_{i}}=U_{\beta_{r}} \cdots$ for all $i$. Since $P\left(U_{\beta_{1}}\right), \ldots, P\left(U_{\beta_{k}}\right)$ are the vertices of a cycle, we can cyclically permute $\mathbb{T}$ and still satisfy $(*)$ and therefore we can assume that $U_{\beta_{i}}=$ $U_{\beta_{1}} \cdots$ for all $i$. Suppose that $\left(t, \varepsilon_{t}\right)=P\left(U_{\beta_{1}}\right) \cap P\left(U_{\beta_{2}}\right)$. We will show that $U_{\beta_{i}}=$ $U_{\beta_{1}} a_{t}^{\varepsilon_{t}} \cdots$ for all $2 \leq i \leq k$. Since $\left(t, \varepsilon_{t}\right)$ is the intersection of $P\left(U_{\beta_{1}}\right)$ and $P\left(U_{\beta_{2}}\right)$, then $X_{t}^{\varepsilon_{t}}=U_{\beta_{1}} a_{t}^{\varepsilon_{t}} \cdots=U_{\beta_{2}} a_{t}^{\varepsilon_{t}} \cdots$ and so $U_{\beta_{2}}=U_{\beta_{1}} a_{t}^{\varepsilon_{t}} \cdots$. Suppose that $U_{\beta_{j-1}}=$ $U_{\beta_{1}} a_{t}^{\varepsilon_{t}} \ldots$. Because there exists an edge $e_{(j-1, j)}$, then $P\left(U_{\beta_{j-1}}\right)$ and $P\left(U_{\beta_{j}}\right)$ must intersect and so there is some $X_{p}^{\varepsilon_{p}}=U_{\beta_{j}} a_{p}^{\varepsilon_{p}} \cdots=U_{\beta_{j-1}} a_{p}^{\varepsilon_{p}} \cdots=U_{\beta_{1}} a_{t}^{\varepsilon_{t}} \cdots a_{p}^{\varepsilon_{p}} \cdots$. Since $\left|U_{\beta_{1}}\right| \leq\left|U_{\beta_{j}}\right|$, then $U_{\beta_{j}}=U_{\beta_{1}} a^{\varepsilon_{t}} a_{t} \cdots$. In particular, $U_{\beta_{k}}=U_{\beta_{1}} a_{t}^{\varepsilon_{t}} \cdots$. Since $P_{\beta_{1}}$ and $P_{\beta_{k}}$ also intersect, there is some $X_{q}^{\varepsilon_{q}}=U_{\beta_{1}} a_{q}^{\varepsilon_{q}} \cdots=U_{\beta_{k}} a_{q}^{\varepsilon_{q}} \cdots$ and so $U_{\beta_{k}}=U_{\beta_{1}} a_{q}^{\varepsilon_{q}} \cdots$. This implies that $\left(t, \varepsilon_{t}\right)=\left(q, \varepsilon_{q}\right)$ and so $P\left(U_{\beta_{1}}\right) \cap P\left(U_{\beta_{2}}\right)=$ $P\left(U_{\beta_{1}}\right) \cap P\left(U_{\beta_{k}}\right)$. However, this is prevented from happening by the condition (**) on the edges and so $\Gamma$ contains no cycles.

Therefore, $\Gamma$ can be written then as a disjoint union of trees. Since $\Gamma$ has $m_{\alpha}$ vertices, then it has at most $m_{\alpha}-1$ edges. By Claim 5.8, each edge corresponds to a single point of intersection of the $P\left(U_{\alpha_{i}}\right)$ and by $(* *)$, there is exactly one edge for each point of intersection. Therefore there are at most $m_{\alpha}-1$ distinct points of intersection and so

$$
\left|\mathbb{S}_{\alpha}\right| \geq \sum_{1 \leq i \leq m}\left|P\left(U_{\alpha_{i}}\right)\right|-\left(m_{\alpha}-1\right) .
$$

Proof of Lemma 5.4. Under a homotopy, there is a one-to-one correspondence between essential fixed point classes which preserves index [J1]. Since $-J$ is the sum 
of the indexes of all the negative fixed point classes, we may then apply the simplification (S1) of Section 2 without changing $J, c^{-}$or $n$. Therefore, we may assume that $X_{i}=U \bar{X}_{i} U^{-1}$ for all $i$ only if $U=1$.

Suppose we have $N$ disjoint sets $\mathbb{S}_{1}, \ldots, \mathbb{S}_{N}$ which satisfy (1)-(3) of Claim 5.9. If $\mathbb{S}_{\alpha}=P\left(U_{\alpha_{1}}\right) \cup \cdots \cup P\left(U_{\alpha_{m_{\alpha}}}\right)$, then by Claims 5.11 and 5.12 ,

$$
\begin{gathered}
\left|\bigcup_{1 \leq \alpha \leq N} \mathbb{S}_{\alpha}\right|=\sum_{1 \leq \alpha \leq N}\left|\mathbb{S}_{\alpha}\right| \geq \sum_{1 \leq i \leq M}\left|P\left(U_{i}\right)\right|-\sum_{1 \leq \alpha \leq N}\left(m_{\alpha}-1\right) \\
\geq\left(J+M-c^{-}+K\right)-(M-N)=J-c^{-}+N+K .
\end{gathered}
$$

Since $\bigcup_{1 \leq j \leq N} \mathbb{S}_{j}=\bigcup_{1 \leq i \leq M} P\left(U_{i}\right)$ is the set of pairs of the form $\left(i, \varepsilon_{i}\right)$ where $1 \leq i \leq n$ and $\varepsilon_{i}=1$ or -1 , then $\left|\bigcup_{j} \mathbb{S}_{j}\right| \leq 2 n$. Therefore, $2 n \geq J-c^{-}+N+K$ and so

$$
J \leq 2 n+c^{-}-(N+K) \leq 2 n+c^{-}-1 .
$$

We will show that we cannot get equality.

If $J=2 n+c^{-}-1$, then

$$
\begin{aligned}
2 n & \geq\left|\bigcup_{1 \leq j \leq N} \mathbb{S}_{j}\right| \geq J-c^{-}+N+K \\
& =\left(2 n+c^{-}-1\right)-c^{-}+N+K=2 n+N+K-1
\end{aligned}
$$

where $N \geq 1$ and $K \geq 0$. Therefore, $N=1, K=0$ and $\left|\bigcup_{i} P\left(U_{i}\right)\right|=2 n$. In other words, all the $P\left(U_{i}\right)$ combine into one set $\mathbb{S}_{1}$ and so there is only one negative fixed point class $F$. Claim 5.10 implies that if $U_{k}$ is the shortest of all the $U_{i}$, then $U_{i}=U_{k} \cdots$. Since $\left|\bigcup_{i} P\left(U_{i}\right)\right|=2 n$, then for every $1 \leq r \leq n$, there is some $i$ and $j$ such that $(r, 1) \in P\left(U_{i}\right)$ and $(r,-1) \in P\left(U_{j}\right)$. Therefore $X_{r}=U_{i} \cdots U_{j}^{-1}=U_{k} \cdots U_{k}^{-1}$ for every $1 \leq r \leq n$. By the assumption at the beginning of this proof, $U_{k}=1$ and so $T(1) \subset F$. However, $x_{0} \in T(1) \subset F$ is a positive fixed point which is only contained in $T(1)$ and so by definition, $K \geq 1$, a contradiction.

\section{REFERENCES}

[B] R. Brown, The Lefschetz Fixed Point Theorem, Scott-Foresman, Glenview, IL, 1971. MR 44:1023

[DHT] O. Davey, E. Hart and K. Trapp, Computation of Nielsen numbers for maps of closed surfaces, Trans. Amer. Math. Soc. 348 (1996), 3245-3266. MR 97g:55001

[J1] B. J. Jiang, Lectures on Nielsen fixed point theory, Contemporary Mathematics 14 (1983). MR 84f:55002

[J2] - Fixed points and braids, I, Invent. Math. 75 (1984), 69-74. MR 85h:55003

[J3] — Fixed points and braids, II, Math. Ann. 272 (1985), 249-256. MR 86i:55004

[J4] Fixed points of surface homeomorphisms, Bull. Amer. Math. Soc. 5 (1981), 176178. MR 82m:55005

[JG] B. Jiang and J. Guo, Fixed points of surface diffeomorphisms, Pacific J. Math. 160 (1993), 67-89. MR 94d:57037

[Ki] T. Kiang, The Theory of Fixed Point Classes, Springer-Verlag, Berlin-New York, 1989. MR 90h:55002

[Mc] C. McCord, Computing Nielsen Numbers, Nielsen Theory and Dynamical Systems, Contemporary Mathematics 152 (1993), 249-267. MR 94e:55004 
[N] J. Nielsen, Über die Minimalzahl der Fixpunkte bei Abbildungstypen der Ringflächen, Math. Ann. 82 (1921), 83-93.

[W] F. Wecken, Fixpunktklassen, III, Math. Ann. 118 (1942), 544-577. MR 5:275b

[We] J. Weier, Über Probleme aus der Topologie der Ebene und der Flächen, Math. Japon. 4 (1956), 101-105. MR 20:4269

Department of Mathematics, California State University, Long Beach, California 90840

E-mail address: pslavich@aol.com 